\title{
SUR DES PROBLÈMES D'ASSERVISSEMENTS STRATIGRAPHIQUES *
}

\author{
GÉrard Gagneux ${ }^{1}$ et Guy Vallet ${ }^{1}$
}

\begin{abstract}
New stratigraphic modellings, developed by the Institut Français du Pétrole, lead to mathematical questions difficult to answer. Such models describe erosion-sedimentation processes and take into account a limited weathering via non standard unilateral problems. Various theoretical results and research procedures are presented for solving the monolithologic column case.
\end{abstract}

Résumé. On expose les difficultés d'ordre mathématique que posent des modèles récents de sédimentation-érosion de bassins élaborés par l'Institut Français du Pétrole et fondés sur la prise en compte de diverses contraintes d'unilatéralité. On présente quelques résultats partiels théoriques et des directions de recherche pour la résolution d'un problème inverse posé par l'étude stratigraphique d'une colonne monolithologique.

Classification Mathématique. 35K20, 35K85, 35Q72.

Reçu le 12 novembre 2001.

\section{Introduction. Formulation du PROBLÈme}

\subsection{Présentation succincte du modèle physique}

Les modèles analysés dans cette étude ont été élaborés au sein de l'Institut Français du Pétrole ( $c f .[4])$ et concernent l'évolution dans un bassin sédimentaire des strates multilithologiques sous la contrainte d'un taux d'érosion maximal dépendant de la composition du mélange et de l'environnement climatique.

On sait que les inéquations d'évolution ont été introduites en 1967 par J.-L. Lions et G. Stampacchia [10] à la suite des remarques liminaires publiées en 1966 (J.-L. Lions [7]) et que leurs applications en Mécanique et en Physique ont semble-t-il été étudiées pour la première fois en 1971 dans le livre fondateur de G. Duvaut et J.-L. Lions [3]. En hommage au professeur J.-L. Lions, on tire de son enseignement et de ses idées matière à fournir ici des outils puissants pour aborder ces problèmes nouveaux.

Un modèle stratigraphique rend compte du transport de sédiments érodables dans un bassin sédimentaire connaissant la tectonique, l'épirogénèse (soulèvement ou affaissement d'ensemble affectant une partie de l'écorce

Mots-clés et phrases : Stratigraphic modelling, variational inequalities, inverse problem, limited weathering.

* Étude menée dans le cadre de la convention scientifique U.P.P.A. (ERS-CNRS 2055) - I.F.P. No. 26386.

1 Laboratoire de Mathématiques Appliquées, Université de Pau et des Pays de l'Adour, BP. 1155, 64013 Pau Cedex, France; e-mail : guy.vallet@univ-pau.fr. 
terrestre), l'eustatisme (i.e. les variations lentes du niveau de la mer, ce qui introduit des constantes physiques de diffusion différentes selon que le point considéré est situé en milieu continental ou marin) et les flux de sédiments aux frontières du bassin. Le transport des sédiments a lieu uniquement en surface : les flux entrants sur une partie du bord sont connus alors que les flux sortants sur la partie complémentaire sont régis par une contrainte unilatérale en spécifiant le flux sortant maximal. La modélisation retenue a pour objet de rendre compatibles un processus de transport gravitaire pour la sédimentation et l'érosion et un mécanisme couplé d'érosion asservi. Le couplage des deux modèles s'articule sur l'introduction d'un facteur variable $\lambda$ (à choisir « au mieux », évoluant dans l'intervalle $[0,1]$ de façon à limiter les flux de matière) et la prise en compte de conditions d'unilatéralité liant la double contrainte du taux d'érosion maximal et de la valeur maximale de $\lambda$.

Le problème de géologie (pétrographie) concerne donc un modèle de sédimentation-érosion de bassins à plusieurs lithologies de porosité supposée constante (les phénomènes de compaction ne sont pas pris en compte pour sérier les difficultés); ses caractéristiques essentielles sont les suivantes :

- le flux de matière est proportionnel au gradient de la hauteur des sédiments déposés;

- une vitesse limite d'érosion est prise en compte (modèle « weather limited »);

- une contrainte d'asservissement instantané régit les variations de l'érosion à l'intérieur du domaine et sur une partie fixe de la frontière.

\subsection{Le problème-modèle simplifié}

\subsubsection{Le problème préparatoire}

Pour mettre en lumière les difficultés d'ordre mathématique que présentent ces modèles nouveaux, on convient d'analyser la situation simplifiée d'une colonne monolithologique.

On note $\Omega \subset \mathbf{R}^{2}$ ou éventuellement $\Omega \subset \mathbf{R}$ la base du bassin, $\left.Q=\Omega \times\right] 0, T[, h(x, t)$ la hauteur de sédiments déposés à l'instant $t$, au-dessus de $x \in \Omega$. Les fonctions inconnues $(x, t) \mapsto(\lambda(x, t), h(t, x))$ sont régies par les relations de conservation, de flux, de Cauchy et les conditions d'unilatéralité suivantes :

$$
\begin{gathered}
L_{0} h \equiv \partial_{t} h-\nabla \cdot(\lambda(x, t) \nabla h)=0 \quad \text { dans } Q, \\
L_{1} h \equiv \partial_{t} h+E(x, t) \geq 0 \quad \text { dans } Q, \\
\lambda(x, t) \in[a, 1], a \geq 0 \quad \text { dans } Q, \\
h(x, 0)=h_{0}(x), \quad x \in \Omega, \\
L_{2}(h, \lambda) \equiv \lambda \partial_{n} h+f \geq 0, L_{1} h \equiv \partial_{t} h+E \geq 0, L_{2}(h, \lambda) \cdot L_{1} h \equiv\left(\lambda \partial_{n} h+f\right)\left(\partial_{t} h+E\right)=0, \\
\operatorname{sur} \Gamma_{s T}=\Gamma_{s} \times[0, T], \quad \Gamma_{s} \cup \Gamma_{e}=\partial \Omega, \\
L_{2}(h, \lambda) \equiv \lambda \partial_{n} h+f=0, \quad \operatorname{sur} \Gamma_{e T}=\Gamma_{e} \times[0, T], \Gamma=\Gamma_{e} \cup \Gamma_{s}=\partial \Omega .
\end{gathered}
$$

Selon Granjean et al. [5], ce modèle « weather limited » limite l'érosion par la contrainte (2) sur le taux d'érosion, où $E$ dépend de l'environnement climatique, pour traduire le fait que les sédiments ne peuvent être érodés que s'ils ont été préalablement attaqués par l'environnement ambiant, ce qui s'exprime ici par la prise en compte d'un taux maximal d'érosion. 


\subsubsection{Questions et conjectures}

Sous des hypothèses raisonnables sur le choix de $\lambda$ considéré momentanément comme un paramètre, on montre en adaptant le chapitre 2 du livre de G. Duvaut et J.-L. Lions [3], (relatif aux problèmes d'asservissements thermiques approchés par un procédé de régularisation hyperbolique du second ordre par rapport à la variable de temps) que le problème $(1,3-5)$ et (6) est bien posé au sens de Hadamard dans un cadre fonctionnel approprié. La difficulté technique réside dans le fait que le paramètre $\lambda$ dépend du temps, ce qui complique notablement l'obtention d'estimations a priori. Dès lors, on peut considérer en première approche (cela sera précisé à la Sect. 4) l'application qui à $\lambda$ fait correspondre $h$ dans des espaces fonctionnels ad hoc.

\section{Deux modélisations sont alors examinées :}

$\mathbf{P b} \mathbf{I})$ Existe-t-il une « solution maximale » $(x, t) \mapsto\left(\lambda^{*}(x, t), h^{*}(x, t)\right)$ au sens où, si l'on note

$$
\Lambda_{a d}=\left\{\lambda \in L^{\infty}(Q),(1)-(6) \text { admet une solution } h\right\},
$$

alors,

$$
\lambda^{*} \in \Lambda_{a d} \quad \text { et } \quad \forall \lambda \in \Lambda_{a d}, \quad \lambda^{*}(x, t) \geq \lambda(x, t), \quad p . p \text {. dans } Q ?
$$

Dans le cas très simplifié où $\lambda$ est stationnaire et l'obstacle $E$ est constant, pris égal à $E_{o}(c f$. Antontsev et al. [1]), la question amène à trouver la fonction $x \mapsto \lambda(x)=\lambda(x, 0)$, la plus grande possible, a priori dans un espace de type fonctions à variation bornée $B V(\Omega)$, solution du problème hyperbolique du premier ordre suivant :

$$
\operatorname{div}\left(\lambda(x) \nabla h_{0}(x)\right)+E_{o} \geq 0, x \in \Omega, \text { au sens des mesures sur } \Omega,
$$

associé à certaines contraintes de flux initial sur le bord.

Ce problème avec un second membre imposé a donné lieu à diverses études fournissant des conditions suffisantes utiles à la notion d'identifiabilité du paramètre $\lambda$ (avec observations distribuées, ponctuelles ou frontières) ; citons en particulier Richter [13], Ito et Kunisch [6], Perez [12]. Ici, la situation est assez différente puisque seul le signe du second membre est imposé et que l'on cherche une solution $\lambda$ maximale dans un ensemble admissible à préciser. On sait à la lumière de ces travaux que l'on sera conduit à faire des hypothèses supplémentaires sur l'état initial via $\nabla h_{0}$ et $\Delta h_{0}$.

Pb II) Peut-on choisir le couple $(x, t) \mapsto(\lambda(x, t), h(x, t))$ de sorte que soit en outre vérifiée plus précisément la contrainte instantanée globale d'asservissement

$$
L_{3} \lambda \equiv 1-\lambda \geq 0, \quad L_{1} h \equiv \partial_{t} h+E \geq 0, \quad L_{3} \lambda \cdot L_{1} h \equiv(1-\lambda)\left(\partial_{t} h+E\right)=0 \quad \text { dans } Q ?
$$

Observons que par un principe du maximum, la réalisation de la contrainte (2) est assurée sous les conditions suffisantes suivantes (cf. Antontsev et al. [1]) :

$$
\left(\mathcal{H}_{*}\right) \begin{cases}\operatorname{div}\left(\partial_{t} \lambda \nabla h\right)+\partial_{t} E-\operatorname{div}(\lambda \nabla E) \geq 0 & \text { dans } Q, \\ \operatorname{div}\left(\lambda(x, 0) \nabla h_{0}(x)\right)+E(x, 0) \geq 0, & x \in \Omega, \\ \partial_{t} f-\partial_{t} \lambda \partial_{n} h+\lambda \partial_{n} E \geq 0 & \text { sur } \Gamma_{e T}=\Gamma_{e} \times[0, T], \\ \lambda \partial_{n} h_{0}+f(0)=0 & \text { sur } \Gamma_{T}=\Gamma \times[0, T] .\end{cases}
$$

Lorsque $\lambda$ et $f$ sont supposés stationnaires et $E$ est pris constant, les relations $\left(\mathcal{H}_{*}\right)$ se résument à la relation $\left(\mathcal{R}_{0}\right)$, outre la condition sur le flux pariétal initial. 


\section{Approche heuristique Sur quelques CAS Simples}

\subsection{Un problème mal posé au sens de Hadamard}

On peut se convaincre que le problème II n'est pas toujours bien posé au sens de Hadamard en examinant le cas particulier trivial où il ne se passe strictement rien! On prend

$$
E=0, f=0 \text { et } h_{0} \text { une constante strictement positive. }
$$

Alors, tout couple de la forme $\left(\lambda, h_{0}\right)$, avec $\lambda \in L^{\infty}(Q), 0 \leq \lambda \leq 1 p . p$. dans $Q$, est solution, ce qui amène probablement la nécessité de définir une solution relative au choix maximal de $\lambda$, ici le couple $\left(1, h_{0}\right)$.

Considérant la même situation mais en relevant l'obstacle $E$ à la valeur constante $E_{0}>0$, on observe que le couple $\left(1, h_{0}\right)$ est encore solution mais il est le seul de la famille précédente; en outre, on sait classiquement $(c f$. G. Duvaut et J.-L. Lions [3], Chap. 2 et diverses généralisations et la Sect. 4) que le seul problème d'asservissement sur le bord relatif à toute donnée de $\lambda$ dans $W^{1, \infty}\left(0, T ; L^{\infty}(\Omega)\right)$ vérifiant ici $0 \leq \lambda \leq 1 p \cdot p$. dans $Q$, admet au plus une solution qui, de fait, est $\left(\lambda, h_{0}\right)$; un tel couple vérifie la contrainte de type $\partial_{t} h+E(x, t) \geq 0$ dans $Q$, mais la réalisation de la contrainte supplémentaire

$$
L_{3} \lambda \equiv 1-\lambda \geq 0, \quad L_{1} h \equiv \partial_{t} h+E_{0} \geq 0, \quad L_{3} \lambda \cdot L_{1} h \equiv(1-\lambda)\left(\partial_{t} h+E_{0}\right)=0 \quad \text { dans } Q
$$

requiert que $\lambda=1$ partout. Dans ce cas, $\left(1, h_{0}\right)$ est donc l'unique solution du problème II.

On construira à la section 3.3.2 des exemples non triviaux de non-unicité qui portent à penser qu'à l'instar de la formulation du problème I, le concept pertinent est celui d'une solution relative à la valeur $\lambda$ maximale.

À cet effet, examinons la situation suivante, d'analyse plus subtile :

On prend $E=0, f=0$ et $h_{0}$ une fonction étagée strictement positive et non constante.

On vérifie que tout couple de la forme $\left(\lambda, h_{0}\right)$, avec $\lambda \in \mathcal{C}(\bar{\Omega}), 0 \leq \lambda \leq 1$ dans $\Omega$ et nulle en les points de discontinuité (dans le cas où $n=1$ ) ou le long des courbes de discontinuité supposées régulières (dans le cas bidimensionnel) est solution : en effet, au sens des mesures, le produit $\lambda \nabla h_{0}$ est nul; cette famille de solutions n'admet pas d'élément maximal. Une éventuelle solution maximale serait donc égale à $1, \mathcal{H}^{n-1}$ presque partout. La question est donc finalement de savoir si l'on peut trouver un couple-solution relatif à la donnée $\lambda=1, \quad i . e$, on est ramené à l'étude du problème d'asservissement rencontré généralement en thermique ( $c f$. G. Duvaut et J.-L. Lions [3], Chap. 2)

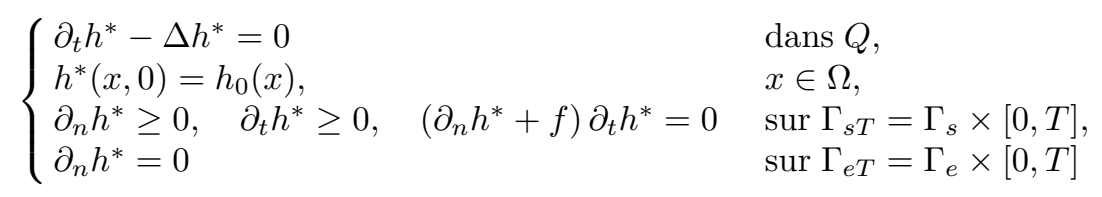

qui admet au plus une solution $h^{*}$ et la question se pose de savoir si cette solution vérifie de plus $\partial_{t} h^{*} \geq 0$ dans $Q$. Il faut observer que $h^{*}$ est nécessairement distincte de $h_{0}$ puisque $h_{0}$ n'est pas harmonique. Il y a là une alternative : on a mis en évidence sur ce cas une infinité de solutions; ou bien, $\left(1, h^{*}\right)$ est solution et de facto solution maximale alors qu'il existe des solutions étrangères au problème (les couples $\left(\lambda, h_{0}\right)$ avec $\lambda$ spécifié plus haut et $h_{0} \neq h^{*}$, ou bien il n'existe pas de solution maximale. On peut conjecturer que la réponse est négative; en effet, la définition d'une solution faible, voire ultra-faible, implique que la fonction $t \mapsto h^{*}(t,$.$) ,$ au voisinage de $t=0$, soit continue au sens des distributions de $\mathcal{D}^{\prime}(\Omega)$. Alors, la contrainte $\partial_{t} h^{*} \geq 0$ dans $Q$ supposée satisfaite, associée à l'équation de continuité du type de l'équation de la chaleur, induit que

$$
h^{*} \in \mathcal{C}^{\infty}(Q) \text { et pour } t>0, \quad \Delta h^{*}(t, .) \geq 0 \text { dans } \Omega
$$


Par continuité des opérateurs de dérivations dans $\mathcal{D}^{\prime}(\Omega)$, il s'ensuit, à la limite, par continuité lorsque $t$ tend vers $0^{+}$, que

$$
\Delta h_{0} \geq 0 \text { au sens des distributions de } \mathcal{D}^{\prime}(\Omega)
$$

et donc aussi au sens des mesures sur $\Omega$; une contradiction en résulte puisque la mesure $\Delta h_{0}$ dans ce choix de figure n'est pas signée. Donc, pour ce cas pathologique probablement hors des réalités géologiques, le problème I n'admet pas de solution maximale au sens précédemment défini mais l'objection trouve ses limites dans le fait que toutes les valeurs de $\lambda$ admissibles concourent à la même solution en $h$.

Examinons alors un cas intermédiaire pour lequel l'état initial ne présente pas de discontinuités.

On prend $\Omega=] 0,1\left[, E=0, f=0\right.$ sur $\partial \Omega, h_{0}$ une fonction continue, affine par morceaux, strictement positive et plate aux extrémités.

On vérifie que tout couple de la forme $\left(\lambda, h_{0}\right)$, avec $\lambda \in L^{\infty}(Q), 0 \leq \lambda \leq 1, \lambda$ étant une fonction étagée valant 0 sur $\{x \in] 0,1\left[, \nabla h_{0} \neq 0\right\}$, est solution. Cette famille contient un élément maximal $\left(\lambda^{*}, h_{0}\right)$, avec :

$$
\lambda^{*}=\chi_{\{x \in] 0,1\left[, \nabla h_{0}=0\right\}}
$$

La question à débattre est donc : peut-on trouver d'autres solutions qui fourniraient l'éventuelle solution maximale hors de cette famille? On observe que nécessairement, on aurait

$$
\lambda(x, t)=1 \quad \text { sur }\{x \in] 0,1\left[, \nabla h_{0}=0\right\} .
$$

Enfin, examinons la curiosité suivante : on prend l'obstacle $E$ égal à la valeur constante $E_{0}>0$ et

$$
f \text { stationnaire, } f \in L^{\infty}(\Gamma), \quad f<0 \text { sur } \Gamma_{e}, \quad f>0 \text { sur } \Gamma_{s},
$$

et pour état initial, une des solutions, à une constante additive près, du problème elliptique

$$
\begin{aligned}
-\Delta h_{0} & =E_{0} \quad \text { dans } \Omega, \\
\partial_{n} h_{0 \mid \Gamma_{e}} & =-f>0, \quad \partial_{n} h_{0 \mid \Gamma_{s}}=g<0, \quad g \in L^{\infty}\left(\Gamma_{s}\right),
\end{aligned}
$$

$g$ vérifiant les seules conditions de compatibilité (ce qui permet de multiples choix)

$$
f+g \geq 0 \quad \text { sur } \Gamma_{s}, \quad \int_{\Gamma_{e}} f \mathrm{~d} \Gamma=\int_{\Gamma_{s}} g \mathrm{~d} \Gamma+E_{0} \operatorname{mes}(\Omega) .
$$

Alors, le couple $\left(1, h: h(\underset{\sim}{x}, t)=-E_{0} t+h_{0}(x)\right)$ est solution ; comme on sait vérifier qu'il ne peut exister d'autres solutions de la forme $(1, \widetilde{h}), \widetilde{h} \neq h$, cette solution est ou bien la solution, si on peut prouver l'unicité (question ouverte), ou bien la solution maximale. Dans ce cas, $\lambda$ et $\partial_{t} h$ sont à chaque instant, en tout point, maximaux. Plus généralement, sous les hypothèses et notations précédentes, se donnant $\lambda \in L^{\infty}(\Omega), 0<\lambda_{0} \leq \lambda \leq 1$.p. dans $\Omega$ et considérant une des solutions, à une constante additive près, du problème elliptique

$$
h_{0} \in H^{1}(\Omega) \text { et vérifie } \forall v \in H^{1}(\Omega), \int_{\Omega} \lambda(x) \nabla h_{0} \cdot \nabla v \mathrm{~d} x=\int_{\Omega} E_{0} v \mathrm{~d} x+\int_{\Gamma_{s}} g v \mathrm{~d} \Gamma-\int_{\Gamma_{e}} f v \mathrm{~d} \Gamma,
$$

on observe que le couple $\left(\lambda, h: h(x, t)=-E_{0} t+h_{0}(x)\right)$ est solution.

Dans ce contexte, des exemples pour lesquels le taux maximal d'érosion n'est jamais atteint (et donc, où $\lambda=1$ ) peuvent être construits de la façon suivante : soit $\mu$ un réel, $\mu \in] 0,1[$ et considérant une des solutions positive, à une constante additive près, du problème elliptique

$$
-\Delta h_{0}=\mu E_{0} \text { dans } \Omega, \quad \partial_{n} h_{0 \mid \Gamma}+f=0
$$


vérifiant la seule condition de compatibilité (ce qui permet de multiples choix)

$$
\int_{\Gamma} f \mathrm{~d} \Gamma=\mu E_{0} \text { mes }(\Omega) .
$$

Alors, le couple $\left(1, h: h(x, t)=-\mu E_{0} t+h_{0}(x)\right)$ est solution; comme on sait vérifier qu'il ne peut exister d'autres solutions de la forme $(1, \widetilde{h}), \widetilde{h} \neq h$, cette solution est ou bien la solution, si l'on peut prouver l'unicité (question ouverte), ou bien la solution maximale.

\subsection{Deux scénarios extrêmes}

On commence par exposer un cas relativement particulier mais très éclairant sur les méthodes de troncature et de principe du maximum mises en œuvre. Supposons que les données physiques vérifient les conditions suivantes, faciles à réaliser en pratique :

$$
(\mathcal{H}) \begin{cases}\partial_{t} E-\Delta E \geq 0 & \text { dans } Q=] 0, T[\times \Omega, \\ \partial_{n} E+\partial_{t} f \geq 0, & \text { sur } \Gamma_{e T}=\Gamma_{e} \times[0, T] \\ \Delta h_{0}+E(0) \geq 0 \text { dans } H^{1}(\Omega), \quad-\partial_{n} h_{0}=f(0) & \text { sur } \Gamma\end{cases}
$$

Considérons alors $h^{*}$ l'unique solution du problème d'asservissement ( $c f$. G. Duvaut et J.-L. Lions [3], Chap. 2)

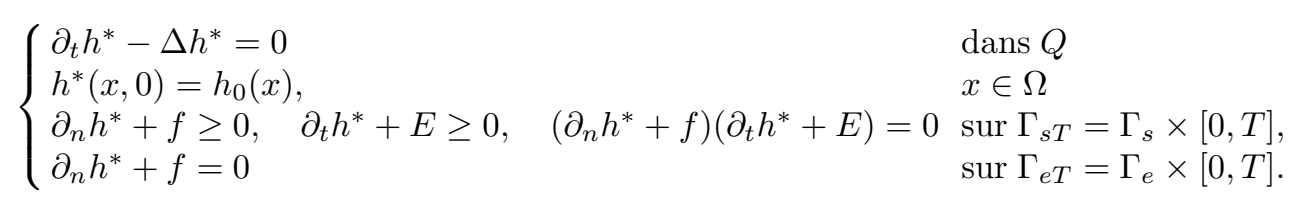

Il est alors facile de vérifier que le couple $\left(1, h^{*}\right)$ est l'unique solution du problème I pour la recherche du coefficient $\lambda$ maximal et une solution pour le problème II d'unilatéralité globale.

Un exemple correspondant à la situation où le taux d'érosion constaté est à chaque instant, en tout point, maximal peut être construit de la façon suivante : on prend

$$
\Omega=] 0,1\left[, \quad E(t, x)=E_{o}>0,\right.
$$

et pour éviter dans un premier temps de fastidieuses discussions, on suppose que les données satisfont les relations :

$$
\begin{gathered}
q_{0}-E_{o} x \geq \partial_{x} h_{0}(x) \text { pour tout } x \in \Omega, \quad E_{0}-q_{0} \leq q_{1} \\
\lambda(0) \partial_{x} h_{0}(0)=-\lambda(0) \partial_{n} h_{0}(0)=q_{0}<0, \quad-\lambda(1) \partial_{x} h_{0}(1)=-\lambda(1) \partial_{n} h_{0}(1) \leq q_{1}, q_{1}>0 .
\end{gathered}
$$

Alors, on établit à la manière de la méthode qui sera détaillée à l'exemple qui suit que le couple $(\lambda, h)$, avec

$$
\lambda(x)=\frac{q_{0}-E_{o} x}{\partial_{x} h_{0}(x)} \quad \text { et } \quad h(x, t)=h_{o}(x)-E_{o} t
$$

est une solution du problème II.

Observons que la situation très particulière pour laquelle l'état initial $h_{0}$ est pris tel que

$$
h_{0}(x)=q_{0} x-\frac{1}{2} E_{o} x^{2}+H, \text { pour tout } x \in \Omega, H \text { réel arbitraire, }
$$

superpose les deux circonstances précédentes puisqu'alors $\lambda$ et $\partial_{t} h$ sont à chaque instant, en tout point, maximaux. 


\subsection{Exemple où $\lambda$ est sous forme tensorielle $\lambda(x, t)=\mathrm{e}^{a(x)} \mathrm{e}^{b(t)}$}

Nous considérons la recherche de solutions particulières a priori de la forme

$$
\lambda(x, t)=\mathrm{e}^{a(x)} \mathrm{e}^{b(t)},\left(\partial_{x} \lambda=a^{\prime}(x) \lambda, \partial_{t} \lambda=b^{\prime}(t) \lambda, \quad \partial_{x t}^{2} \lambda=a^{\prime}(x) b^{\prime}(t) \lambda\right) .
$$

On introduit la nouvelle fonction inconnue

$$
u(x, t)=\partial_{t} h(x, t) \quad(E=0 \text { pour aller à l'essentiel...) }
$$

qui satisfait l'équation suivante :

$$
\left.\partial_{t} u=\partial_{x}\left(\lambda \partial_{x} u+\partial_{x} h \partial_{t} \lambda\right) \equiv \partial_{x}\left(\lambda \partial_{x} u\right)+\partial_{t x}^{2} \lambda \partial_{x} h+\partial_{t} \lambda \partial_{x}^{2} h=0, \quad(x, t) \in Q=\right] 0, T[\times \Omega
$$

ou, utilisant l'équation (1) :

$$
\left.\partial_{t} u=\partial_{x}\left(\lambda \partial_{x} u\right)+A \partial_{x} h+\frac{\partial_{t} \lambda}{\lambda} u, \quad(x, t) \in Q=\right] 0, T[\times \Omega
$$

où l'on a posé

$$
A=\partial_{t x}^{2} \lambda-\frac{\partial_{x} \lambda \partial_{t} \lambda}{\lambda}
$$

et en vertu du calcul (7)

$$
A=\partial_{t x}^{2} \lambda-\frac{\partial_{x} \lambda \partial_{t} \lambda}{\lambda}=a^{\prime} b^{\prime} \lambda-a^{\prime} b^{\prime} \lambda=0, \quad \frac{\partial_{t} \lambda}{\lambda}=b^{\prime}(t)
$$

Consécutivement, nous arrivons à l'équation reformulée

$$
\left.\partial_{t} u=\partial_{x}\left(\lambda \partial_{x} u\right)+b^{\prime}(t) u, \quad(x, t) \in Q=\right] 0, T[\times \Omega,
$$

et donc, introduisant pour ce problème parabolique, la fonction auxiliaire

$$
v(x, t)=\mathrm{e}^{\sigma t} u(x, t)
$$

nous parvenons à l'équation

$$
\left.\partial_{t} v=\partial_{x}\left(\lambda \partial_{x} v\right)+\left(b^{\prime}(t)+\sigma\right) v, \quad(x, t) \in Q=\right] 0, T[\times \Omega .
$$

Choisissant la constante $\sigma$ telle que

$$
\left.b^{\prime}(t)+\sigma \leq 0 \quad \text { pour tout } t \in\right] 0, T[
$$

nous pouvons garantir que

$$
\left.v(x, t)=\mathrm{e}^{\sigma t} u(x, t)=\mathrm{e}^{\sigma t} h_{t}(x, t) \geq 0, \quad(x, t) \in Q=\right] 0, T[\times \Omega
$$

si

$$
v(x, 0)=u(x, 0)=\partial_{t} h(x, 0)=\partial_{x}\left(\lambda(x, 0) \partial_{x} h(x, 0)\right)=\varepsilon^{2}(x) \geq 0,
$$

outre des conditions appropriées sur le bord latéral que nous allons analyser ultérieurement. 
Dans le cas le plus général de la recherche de solutions peu régulières ( $c f$. Antontsev et al. [1]), $\varepsilon^{2}$ représenterait une distribution positive sur ]0,1[, et donc une mesure positive sur ]0, 1[. D'après un résultat de Schwartz ([14], pp. 29, 53-54) la fonction $x \mapsto \lambda(x, 0) \partial_{x} h_{0}(x)$ admet dans sa classe de Lebesgue un représentant borné et croissant au sens large.

De plus, $\partial_{t} h$, s'il n'est pas pris constant, ne peut atteindre son minimum que sur la frontière parabolique du cylindre $Q$. Par exemple, si nous considérons la condition de frontière

$$
L_{2}(h, \lambda) \equiv \lambda \partial_{x} h+f=0, \quad \operatorname{sur} \Gamma_{e T}=\{0\} \times[0, T], \Gamma=\Gamma_{e} \cup \Gamma_{s}=\partial \Omega
$$

avec $f=0$, alors

$$
L_{2}(h, \lambda) \equiv \lambda \partial_{x} h=0 \Rightarrow \partial_{t x} h(0, t)=\partial_{x} u(0, t)=\partial_{x} v(0, t)=0, \text { sur } \Gamma_{e T}=\{0\} \times[0, T] .
$$

En conséquence de ce principe du minimum, la résolution du problème II par cette voie est sans issue car alors, les conditions d'unilatéralité globale impliqueraient que $\lambda \equiv 1$ et $\partial_{t} h>0$ dans $Q$ et donc, $a$ et $b$ seraient identiquement nulles. On s'intéresse par la force des choses au problème I!

On doit en outre veiller à ce que

$$
\begin{gathered}
L_{2}(h, \lambda) \equiv \lambda \partial_{x} h+q_{1}(t) \geq 0, \quad L_{1} \equiv u \geq 0, \quad L_{2} L_{1} \equiv\left(\lambda \partial_{x} h+q_{1}(t)\right) u=0, \\
\operatorname{sur} \Gamma_{s T}=\{1\} \times[0, T], \Gamma_{s} \cup \Gamma_{e}=\partial \Omega,
\end{gathered}
$$

cependant qu'on pose la condition de flux entrant sous la forme

$$
-\lambda(0, t) \partial_{x} h(0, t)+q_{0}(t)=0, \quad t \geq 0
$$

et qu'on impose la condition de flux sortant, pour satisfaire (18)

$$
\lambda(1, t) \partial_{x} h(1, t)+q_{1}(t)=0, \quad t \geq 0
$$

ce qui suppose en particulier que les données du modèle soient telles que

$$
\left\{\frac{q_{0}(0)}{\partial_{x} h_{0}(0)}, \frac{-q_{1}(1)}{\partial_{x} h_{0}(1)}\right\} \in\left[\lambda_{1}, \lambda_{2}\right]^{2} .
$$

Alors nous avons à trouver une solution du problème $(13,15-18)$ telle que

$$
u(x, t)=\partial_{t} h(x, t) \geq 0 \quad \text { dans } Q
$$

avec des fonctions $a(x), b(t)$ convenables et négatives assurant de fait que

$$
\left.\lambda(x, t)=\mathrm{e}^{a(x)} \mathrm{e}^{b(t)} \in\left[a_{0}, 1\right] \subset\right] 0,1[\operatorname{dans} Q .
$$

Dès lors, l'équation (15) définit, en se reportant à Antontsev et al. [1] pour les détails du traitement de la solution $\lambda^{*}$ maximale, la fonction $x \mapsto a(x)$ par

$$
\lambda(x, 0)=\mathrm{e}^{a(x)} \mathrm{e}^{b(0)}=\frac{\int_{0}^{x} \mathrm{~d} \varepsilon^{2}(s)+q_{0}(0)}{\partial_{x} h_{0}(x)}, \quad \mathrm{e}^{a(x)}=\mathrm{e}^{-b(0)} \frac{\int_{0}^{x} \mathrm{~d} \varepsilon^{2}(s)+q_{0}(0)}{\partial_{x} h_{0}(x)}
$$


et, consécutivement, la fonction $\lambda$ grâce aux relations

$$
\begin{aligned}
& \lambda(x, t)=\mathrm{e}^{a(x)} \mathrm{e}^{b(t)}=\mathrm{e}^{b(t)} \mathrm{e}^{-b(0)} \frac{\int_{0}^{x} \mathrm{~d} \varepsilon^{2}(s)+q_{0}(0)}{\partial_{x} h_{0}(x)}, \\
& \lambda(0, t)=\mathrm{e}^{a(0)} \mathrm{e}^{b(t)}=\mathrm{e}^{b(t)} \mathrm{e}^{-b(0)} \frac{q_{0}(0)}{\partial_{x} h_{0}(0)}, \\
& \lambda(1, t)=\mathrm{e}^{a(1)} \mathrm{e}^{b(t)}=\mathrm{e}^{b(t)} \mathrm{e}^{-b(0)} \frac{\int_{0}^{1} \mathrm{~d} \varepsilon^{2}(s)+q_{0}(0)}{\partial_{x} h_{0}(1)}
\end{aligned}
$$

en imposant (condition de cohérence) la valeur de la masse totale de la mesure $\varepsilon^{2}$

$$
\int_{0}^{1} \mathrm{~d} \varepsilon^{2}(s)+q_{0}(0)=-q_{1}(0)<0 \quad \text { et donc } \quad q_{1}(0)+q_{0}(0) \leq 0
$$

ce qui est moral puisqu'ici, par le choix de $E=0$, on recherche une élévation (ou tout au moins, un nonaffaissement) en tout point du niveau au cours du temps.

Des conditions de bord suffisantes $\left(c f\right.$. les relations $\left.\left(\mathcal{H}_{*}\right)\right)$ pour garantir la positivité de $\partial_{t} h$ s'expriment par

$$
\begin{aligned}
\lambda^{\prime}(0, t) \partial_{x} h(0, t)+q_{0}^{\prime}(t) \geq 0 & \text { pour } t \geq 0, \\
-\lambda^{\prime}(1, t) \partial_{x} h(1, t)+q_{1}^{\prime}(t) \geq 0 & \text { pour } t \geq 0,
\end{aligned}
$$

ce qui, compte-tenu des conditions de bord, implique que les fonctions

$$
t \mapsto \lambda(0, t) q_{0}(t) \quad \text { et } \quad t \mapsto \lambda(1, t) q_{1}(t)
$$

sont croissantes.

Il est alors loisible de prendre comme cas simple d'illustration (d'autres choix sont possibles...) la fonction $t \mapsto \lambda(1, t) q_{1}(t)$ constante, ce qui permet de déterminer la fonction $b$ par la relation

$$
\mathrm{e}^{b(t)} \mathrm{e}^{-b(0)}=\frac{q_{1}(0)}{q_{1}(t)}, \quad t \geq 0, \quad \text { avec } \quad q_{1}(t) \geq q_{1}(0) \quad \text { pour } t \geq 0
$$

et donc

$$
\lambda(0, t) q_{0}(t)=\frac{q_{1}(0)}{q_{1}(t)} \frac{q_{0}(0)}{\partial_{x} h_{0}(0)} q_{0}(t) \text { pour } t \geq 0
$$

Dès lors, il suffit de choisir $t \mapsto q_{0}(t)$ et $t \mapsto q_{1}(t)$ de sorte que la fonction $t \mapsto \frac{q_{0}(t)}{q_{1}(t)}$ soit croissante (ce qui peut se faire de multiples manières; par exemple, $q_{0}(t)=q_{0}<0$ et $t \mapsto q_{1}(t)$ croissante et donc, $b^{\prime}(t)=-\frac{q_{1}^{\prime}(t)}{q_{1}(t)}$ et dans les calculs antérieurs, $\sigma=0$ ) pour que toutes les conditions requises soient remplies. 
Il en résulte finalement que $\lambda^{*}$ s'exprime par la formule explicite :

$$
\lambda^{*}(x, t)=\frac{q_{1}(0)}{q_{1}(t)} \frac{\int_{0}^{x} \mathrm{~d} \varepsilon^{2}(s)+q_{0}(0)}{\partial_{x} h_{0}(x)}, \quad(x, t) \in Q,
$$

$x \mapsto \int_{0}^{x} \mathrm{~d} \varepsilon^{2}(s)$ étant à choisir de façon optimale en fonction de la connaissance de $h_{0}$ selon les indications de Antontsev et al. [1].

Une variante facile est obtenue en prenant $t \mapsto \lambda(0, t) q_{0}(t)$ constante et il s'ensuit que l'on a

$$
\lambda^{*}(x, t)=\frac{q_{0}(0)}{q_{0}(t)} \frac{\int_{0}^{x} \mathrm{~d} \varepsilon^{2}(s)+q_{0}(0)}{\partial_{x} h_{0}(x)}, \quad(x, t) \in Q,
$$

sous les hypothèses que

$$
q_{0}(t) \leq q_{0}(0) \leq 0 \quad \text { et } \quad t \rightarrow \frac{q_{0}(t)}{q_{1}(t)} \text { soit croissante. }
$$

Plus généralement, on peut prendre

$$
\lambda(1, t) q_{1}(t)=\lambda(1,0) q_{1}(0)+\Psi(t), \quad t \geq 0, \quad \Psi(0)=0, \Psi \text { croissante }
$$

et de simples calculs conduisent à l'expression

$$
\lambda^{*}(x, t)=\left[\frac{q_{1}(0)}{q_{1}(t)}-\frac{\partial_{x} h_{0}(1)}{q_{1}(0)} \frac{\Psi(t)}{q_{1}(t)}\right] \frac{\int_{0}^{x} \mathrm{~d} \varepsilon^{2}(s)+q_{0}(0)}{\partial_{x} h_{0}(x)}, \quad(x, t) \in Q,
$$

sous l'astreinte que

$$
\frac{q_{1}(0)}{q_{1}(t)}-\frac{\partial_{x} h_{0}(1)}{q_{1}(0)} \frac{\Psi(t)}{q_{1}(t)} \leq 1 \quad \text { et } \quad t \mapsto \frac{q_{0}(t)}{q_{1}(t)}\left[q_{1}(0)-\frac{\partial_{x} h_{0}(1)}{q_{1}(0)} \Psi(t)\right] \text { soit croissante. }
$$

\section{Construction DE SOlUtions PARTiCulières « TRAVELling WAVES 》}

Une question ouverte intéressante concerne le problème de l'élaboration et l'analyse de solutions particulières de l'équation (1) et de ses conditions associées dans le cadre du problème II, dites solutions travelling waves ${ }^{2}$. Il s'agit de chercher des solutions de la forme

$$
h(x, t)=h(\xi), \quad \lambda(x, t)=\lambda(\xi), \quad \xi=\mu x+t \text { ou } \xi=x+\mu t
$$

lorsque $\Omega=] 0, x_{1}[\subset] 0,+\infty\left[, \Gamma_{e}=\{x=0\}, \Gamma_{s}=\left\{x=x_{1}\right\}\right.$ et que les données $h_{0}$ et $q_{0}, q_{1}$ seront choisies de façon ad hoc.

\footnotetext{
${ }^{2}$ Sur une idée de Antontsev, professeur invité à l'Université de Pau en juin 2000 et juin 2001. Les auteurs tiennent à exprimer leur reconnaissance au Pr. Antontsev (Université d'état de Sibérie à Novossibirsk) pour les nombreuses discussions stimulantes qu'il a engagées sur le sujet.
} 


\subsection{Solution spéciale $: h(x, t)=h(\xi), \lambda(x, t)=\lambda(\xi), \xi=\mu x+t$}

\subsubsection{Premier exemple}

On se propose d'examiner ici la possibilité des circonstances enchaînées suivantes :

$$
\begin{gathered}
L_{1} h \equiv h^{\prime}(\xi)+E \geq 0, L_{3} \lambda \equiv 1-\lambda(\xi)=0,0<\xi<\xi_{0} \\
L_{1} h \equiv h^{\prime}(\xi)+E=0, L_{3} \lambda \equiv 1-\lambda(\xi) \geq 0, \xi_{0}<\xi<\xi_{1}<+\infty .
\end{gathered}
$$

Nous avons à trouver des solutions de l'équation de continuité

$$
L_{0} h \equiv \partial_{t} h-\partial_{x}\left(\lambda(x, t) \partial_{x} h\right)=0 \quad \operatorname{dans} Q
$$

de la forme suivante

$$
\begin{gathered}
h(x, t)=h(\xi), \quad \lambda(x, t)=\lambda(\xi), \quad \xi=\mu x+t, \\
\partial_{t} h(x, t)=h^{\prime}(\xi), \quad \partial_{x} h(x, t)=\mu h^{\prime}(\xi) .
\end{gathered}
$$

Ce faisant, nous réduisons la question à l'étude de l'équation différentielle

$$
\left.L_{0} h \equiv h^{\prime}(\xi)-\mu^{2}\left(\lambda(\xi) h^{\prime}(\xi)\right)^{\prime}=0 \quad \text { dans } Q=\right] 0,+\infty[
$$

ou, après une quadrature,

$$
\left.h(\xi)-\mu^{2} \lambda(\xi) h^{\prime}(\xi)=C_{0} \quad \operatorname{dans} Q=\right] 0,+\infty[
$$

ce qui montre que les fonctions $\xi \mapsto \lambda(\xi)$ et $\xi \mapsto h^{\prime}(\xi)$ peuvent éventuellement présenter des discontinuités mais que le produit $\xi \mapsto \lambda(\xi) h^{\prime}(\xi)$ est nécessairement continu.

L'intervalle ]0, $\xi_{0}[$.

Supposons que sur l'intervalle $] 0, \xi_{0}[$, on convienne de réaliser

$$
L_{1} h \equiv h^{\prime}(\xi)+E \geq 0, \quad L_{3} \lambda \equiv 1-\lambda(\xi)=0, \quad 0<\xi<\xi_{0} .
$$

Alors, selon $(20,21)$, nous avons à considérer le problème suivant :

$$
L_{1} h \equiv h^{\prime}(\xi)+E \geq 0, \quad h(\xi)-\mu^{2} \cdot h^{\prime}(\xi)=C_{0}, \quad 0<\xi<\xi_{0} .
$$

Le problème (22) admet des solutions de la forme

$$
h(\xi)=C_{1} \mathrm{e}^{\frac{\xi}{\mu^{2}}}+C_{0}, \quad 0<\xi<\xi_{0}, \quad C_{1} \geq-\mu^{2} E \mathrm{e}^{-\frac{\xi_{0}}{\mu^{2}}}
$$

Nous privilégions pour la suite du scénario la solution telle que

$$
L_{1}\left(h \mid \xi_{0}\right) \equiv h^{\prime}\left(\xi_{0}\right)+E=0
$$

i.e.

$$
\begin{gathered}
h(\xi)=-\mu^{2} E \mathrm{e}^{-\frac{\xi_{0}}{\mu^{2}}} \mathrm{e}^{\frac{\xi}{\mu^{2}}}+\mu^{2} E \mathrm{e}^{-\frac{\xi_{0}}{\mu^{2}}}+h_{0}(0), \quad h_{0}(0)=h(0)>0, \quad 0<\xi<\xi_{0}, \\
L_{3} \lambda \equiv 1-\lambda(\xi)=0, \quad 0<\xi<\xi_{0}
\end{gathered}
$$


$\left(h_{0}(0)=h(0)>0, \xi_{0}>0\right.$ et $\mu$ sont arbitraires) et il est facile de vérifier que

$$
\begin{gathered}
L_{1}(h \mid \xi) \equiv h^{\prime}(\xi)+E=-E \mathrm{e}^{-\frac{\xi_{0}-\xi}{\mu^{2}}}+E=E\left(1-\mathrm{e}^{-\frac{\xi_{0}-\xi}{\mu^{2}}}\right) \geq 0, \quad 0<\xi<\xi_{0} . \\
L_{1}\left(h \mid \xi_{0}\right) \equiv h^{\prime}\left(\xi_{0}\right)+E=E(1-1)=0 .
\end{gathered}
$$

L'intervalle $] \xi_{0},+\infty[$.

À présent, considérons l'intervalle $] \xi_{0},+\infty[$, où nous cherchons à respecter les contraintes

$$
L_{1} h \equiv h^{\prime}(\xi)+E=0, \quad L_{3} \lambda \equiv 1-\lambda(\xi) \geq 0, \quad \xi_{0}<\xi<+\infty .
$$

Alors, selon $(20,25)$, nous avons à considérer le problème suivant :

$$
L_{1} h \equiv h^{\prime}(\xi)+E=0, \quad h(\xi)-\mu^{2} \cdot \lambda h^{\prime}(\xi)=C_{0}, \quad \lambda(\xi) \leq 1, \quad \xi_{0}<\xi<\infty .
$$

Le problème (26) admet une solution donnée par

$$
h(\xi)=-E \xi+E \xi_{0}+h_{0}(0)-\mu^{2} E\left(1-\mathrm{e}^{-\frac{\xi_{0}}{\mu^{2}}}\right), \quad \lambda(\xi)=\frac{\xi}{\mu^{2}}+1-\frac{\xi_{0}}{\mu^{2}}
$$

et on vérifie que

$$
\begin{gathered}
L_{1} h \equiv h^{\prime}(\xi)+E=-E+E=0, \quad \xi_{0}<\xi<+\infty, \\
h\left(\xi_{0}-0\right)=-\mu^{2} E+\mu^{2} E \mathrm{e}^{-\frac{\xi_{0}}{\mu^{2}}}+h_{0}(0)=h\left(\xi_{0}+0\right)=h_{0}(0)-\mu^{2} E\left(1-\mathrm{e}^{-\frac{\xi_{0}}{\mu^{2}}}\right), \\
h\left(\xi_{1}\right)=-E \xi_{1}+E \xi_{0}+h_{0}(0)-\mu^{2} E\left(1-\mathrm{e}^{-\frac{\xi_{0}}{\mu^{2}}}\right)=-E \xi_{1}+E \xi_{0}+h\left(\xi_{0}\right)>0
\end{gathered}
$$

si

$$
\xi_{0}<\xi_{1}<\xi_{0}+\frac{h_{0}(0)}{E}-\mu^{2}\left(1-\mathrm{e}^{-\frac{\xi_{0}}{\mu^{2}}}\right)=\xi_{0}+\frac{h\left(\xi_{0}\right)}{E},
$$

mais on se heurte de fait à la contradiction redhibitoire suivante :

$$
\lambda(\xi)=\frac{\xi}{\mu^{2}}+1-\frac{\xi_{0}}{\mu^{2}}>1 .
$$

Ainsi, par ce procédé, on ne peut construire une solution admissible lorsque l'obstacle est stationnaire et on va donc montrer qu'en relachant la contrainte, c'est-à-dire en relevant la valeur de la vitesse limite d'érosion, on va pouvoir construire une solution convenable sur un intervalle contenu dans ] $\xi_{0},+\infty$ [ et d'autant plus long qu'on aura plus fortement relevé l'obstacle. On considère donc, toute chose étant égale par ailleurs, les contraintes nouvelles

$$
L_{1} h \equiv h^{\prime}(\xi)+E^{*}=0, \quad E^{*}>E, \quad L_{3} \lambda \equiv 1-\lambda(\xi) \geq 0, \quad \xi_{0}<\xi<+\infty .
$$


Reprenant les calculs précédents, la solution trouvée s'exprime par

$$
h(\xi)=-E^{*} \xi+E^{*} \xi_{0}+h_{0}(0)-\mu^{2} E\left(1-\mathrm{e}^{-\frac{\xi_{0}}{\mu^{2}}}\right), \quad \lambda(\xi)=\frac{\xi-\xi_{0}}{\mu^{2}}+\frac{E}{E^{*}}
$$

solution admissible sur tout l'intervalle $] \xi_{0}, \xi_{1}[$ tel que

$$
\frac{\xi_{1}-\xi_{0}}{\mu^{2}}+\frac{E}{E^{*}} \leq 1
$$

En résumé, prenant

$$
E=E \chi_{] 0, \xi_{0}[}+E^{*} \chi_{] \xi_{0}, \xi_{1}[}, \quad E^{*}>E, \quad \xi=\mu x+t
$$

on met en évidence le couple-solution (avec les notations et conditions précédentes)

$$
\begin{aligned}
& h(x, t)=-\mu^{2} E \mathrm{e}^{-\frac{\xi_{0}}{\mu^{2}}} \mathrm{e}^{\frac{\xi}{\mu^{2}}}+\mu^{2} E \mathrm{e}^{-\frac{\xi_{0}}{\mu^{2}}}+h_{0}(0) ; \quad \lambda(x, t)=1, \quad 0<\xi \leq \xi_{0}, \\
& h(x, t)=-E^{*} \xi+E^{*} \xi_{0}+h_{0}-\mu^{2} E\left(1-\mathrm{e}^{-\frac{\xi_{0}}{\mu^{2}}}\right) ; \quad \lambda(x, t)=\frac{\xi-\xi_{0}}{\mu^{2}}+\frac{E}{E^{*}}, \quad \xi_{0}<\xi \leq \xi_{1} .
\end{aligned}
$$

On observe que les fonctions $\xi \mapsto \lambda(\xi)$ et $\xi \mapsto h^{\prime}(\xi)$ sont discontinues puisque

$$
\lambda\left(\xi_{0}-0\right)=1, \quad \lambda\left(\xi_{0}+0\right)=\frac{E}{E^{*}}<1, \quad h^{\prime}\left(\xi_{0}-0\right)=-E, \quad h^{\prime}\left(\xi_{0}-0\right)=-E^{*}
$$

mais que le produit $\lambda h^{\prime}$ est continu.

\subsubsection{Second exemple}

On se propose d'examiner ici la possibilité des circonstances en cascade suivantes :

$$
\begin{gathered}
L_{1} h \equiv h^{\prime}(\xi)+E=0, \quad L_{3} \lambda \equiv 1-\lambda(\xi) \geq 0, \quad 0<\xi<\xi_{0} \\
L_{1} h \equiv h^{\prime}(\xi)+E^{*} \geq 0, \quad L_{3} \lambda \equiv 1-\lambda(\xi)=0, \quad \xi_{0}<\xi<\xi_{1}
\end{gathered}
$$

L'intervalle $] 0, \xi_{0}[$.

À présent, considérons l'intervalle $] 0, \xi_{0}[$, où

$$
L_{1} h \equiv h^{\prime}(\xi)+E=0, \quad L_{3} \lambda \equiv 1-\lambda(\xi) \geq 0, \quad 0<\xi<\xi_{0}
$$

Alors, selon (20), nous avons à considérer le problème suivant

$$
L_{1} h \equiv h^{\prime}(\xi)+E=0, \quad h(\xi)-\mu^{2} \cdot \lambda h^{\prime}(\xi)=C_{0}, \quad \lambda(\xi) \leq 1, \quad 0<\xi<\xi_{0} .
$$

Le problème (31) admet une solution donnée par

$$
\begin{gathered}
h(\xi)=-E \xi+h_{0}(0), \quad \lambda(\xi)=\frac{\xi}{\mu^{2}}+1-\frac{\xi_{0}}{\mu^{2}}<1, \\
\lambda(\xi) \in] 1-\frac{\xi_{0}}{\mu^{2}}, 1\left[\subset \left[0,1\left[\quad \text { si } \frac{\xi_{0}}{\mu^{2}} \leq 1, \quad \lambda\left(\xi_{0}\right)=1,\right.\right.\right. \\
C_{0}=h_{0}(0)+\mu^{2} E\left(1-\frac{\xi_{0}}{\mu^{2}}\right) . \\
h(\xi) \in] h_{0}(0)-E \xi_{0}, h_{0}(0)[\subset] 0, h_{0}(0)\left[\text { si } E \xi_{0}<h_{0}(0) .\right.
\end{gathered}
$$


Alors, nous avons à respecter les conditions de compatibilité suivantes :

$$
E \xi_{0}<h_{0}(0), \quad \xi_{0} \leq \mu^{2} .
$$

L'intervalle $] \xi_{0},+\infty[$.

Supposons que sur l'intervalle $] \xi_{0}, \xi_{1}[$, on cherche à réaliser

$$
L_{1} h \equiv h^{\prime}(\xi)+E^{*} \geq 0, \quad L_{3} \lambda \equiv 1-\lambda(\xi)=0, \quad \xi_{0}<\xi<\xi_{1} .
$$

Alors, nous avons à considérer le problème suivant :

$$
L_{1} h \equiv h^{\prime}(\xi)+E^{*} \geq 0, \quad h(\xi)-\mu^{2} \cdot h^{\prime}(\xi)=C_{0}, \quad \xi_{0}<\xi<\xi_{1} .
$$

L'équation

$$
h(\xi)-\mu^{2} \cdot h^{\prime}(\xi)=C_{0}, \quad \xi_{0}<\xi<\xi_{1}
$$

avec la contrainte d'obstacle admet pour famille de solutions

$$
h(\xi)=C_{1} \mathrm{e}^{\frac{\xi}{\mu^{2}}}+C_{0}, \quad \xi_{0}<\xi<\xi_{1}, \quad \text { avec } \quad C_{1} \geq-\mu^{2} E^{*} \mathrm{e}^{-\frac{\xi 1}{\mu^{2}}} .
$$

Nous retenons la solution

$$
h(\xi)=-\mu^{2} E \mathrm{e}^{-\frac{\xi_{0}-\xi}{\mu^{2}}}+C_{0}=-\mu^{2} E \mathrm{e}^{-\frac{\xi_{0}}{\mu^{2}}} \mathrm{e}^{\frac{\xi}{\mu^{2}}}+\mu^{2} E\left(1-\frac{\xi_{0}}{\mu^{2}}\right)+h_{0}(0), \quad \xi_{0}<\xi<\xi_{1},
$$

de façon à satisfaire la condition de continuité

$$
h\left(\xi_{0}-0\right)=-E \xi_{0}+h_{0}=h\left(\xi_{0}+0\right)=C_{1} \mathrm{e}^{\frac{\xi_{0}}{\mu^{2}}}+C_{0} .
$$

Il s'ensuit donc la condition de compatibilité entre hauteurs des obstacles et durées des phénomènes

$$
\frac{E}{E^{*}} \leq \mathrm{e}^{\frac{\xi_{0}-\xi_{1}}{\mu^{2}}}
$$

et la condition de réalisme donnant la valeur limite de $\xi_{1},\left(h\left(\xi_{1}\right)=0\right)$

$$
h\left(\xi_{1}\right)=-\mu^{2} E \mathrm{e}^{\frac{\xi_{0}-\xi_{1}}{\mu^{2}}}+\mu^{2} E\left(1-\frac{\xi_{0}}{\mu^{2}}\right)+h_{0}(0)=0 .
$$

Il découle des précautions précédentes que

$$
L_{1}(h \mid \xi) \equiv h^{\prime}(\xi)+E^{*}=-E \mathrm{e}^{-\frac{\xi_{0}-\xi}{\mu^{2}}}+E^{*} \geq-E \mathrm{e}^{\frac{\xi_{0}-\xi_{1}}{\mu^{2}}}+E^{*} \geq 0, \quad \xi_{0}<\xi<\xi_{1} .
$$

En résumé, prenant

$$
E(\xi)=E \chi_{] 0, \xi_{0}[}+E^{*} \chi_{] \xi_{0}, \xi_{1}[}, \quad E^{*}>E, \quad \xi=\mu x+t,
$$

on met en évidence le couple-solution (avec les notations et conditions précédentes)

$$
\begin{aligned}
& h(x, t)=-E \xi+h_{0}(0), \quad \lambda(x, t)=\frac{\xi}{\mu^{2}}+1-\frac{\xi_{0}}{\mu^{2}}, \quad 0<\xi \leq \xi_{0}, \\
& h(x, t)=-\mu^{2} E \mathrm{e}^{-\frac{\xi_{0}}{\mu^{2}}} \mathrm{e}^{\frac{\xi}{\mu^{2}}}+\mu^{2} E\left(1-\frac{\xi_{0}}{\mu^{2}}\right)+h_{0}(0), \quad \lambda(x, t)=1, \quad \xi_{0} \leq \xi \leq \xi_{1} .
\end{aligned}
$$




\subsection{Solution spéciale $: h(x, t)=h(\xi), \lambda(x, t)=\lambda(\xi), \xi=x+\mu t$}

On se propose d'examiner ici la possibilité des circonstances successives suivantes :

$$
\begin{gathered}
h=h(\xi), \lambda=\lambda(\xi), \xi=x+\mu t, \\
L_{1} h \equiv \mu h^{\prime}(\xi)+E \geq 0, L_{3} \lambda \equiv 1-\lambda(\xi)=0,0<\xi<\xi_{0} \\
L_{1} h \equiv \mu h^{\prime}(\xi)+E^{*}=0, \quad E^{*}>E, \quad L_{3} \lambda \equiv 1-\lambda(\xi) \geq 0, \xi_{0}<\xi<+\infty .
\end{gathered}
$$

Il y a donc à réaliser les conditions suivantes :

$$
\begin{gathered}
h(x, t)=h(\xi), \quad \lambda(x, t)=\lambda(\xi), \quad \xi=x+\mu t, \\
\partial_{t} h(x, t)=\mu h^{\prime}(\xi), \quad \partial_{x} h(x, t)=h^{\prime}(\xi) \\
\left.L_{0} h \equiv \mu h^{\prime}(\xi)-\left(\lambda(\xi) h^{\prime}(\xi)\right)^{\prime}=0 \quad \text { dans } Q=\right] 0,+\infty[ \\
L_{1} h \equiv \mu h^{\prime}(\xi)+E \geq 0, \quad L_{3} \lambda \equiv 1-\lambda(\xi)=0, \quad 0<\xi<\xi_{0}, \\
L_{1} h \equiv \mu h^{\prime}(\xi)+E^{*}=0, \quad L_{3} \lambda \equiv 1-\lambda(\xi) \geq 0, \quad \xi_{0}<\xi<+\infty \\
L_{1} L_{3} \equiv\left(\mu h^{\prime}(\xi)+E\right)(1-\lambda(\xi))=0, \quad 0<\xi<+\infty . \\
\left.\mu h(\xi)-\lambda(\xi) h^{\prime}(\xi)=C_{0} \quad \text { dans } Q=\right] 0,+\infty[, \text { après une quadrature. }
\end{gathered}
$$

3.2.1. L'intervalle $] 0, \xi_{0}[$

Supposons que sur l'intervalle $] 0, \xi_{0}[$ on veuille réaliser

$$
L_{1} h \equiv \mu h^{\prime}(\xi)+E \geq 0, \quad L_{3 \lambda} \equiv 1-\lambda(\xi)=0, \quad 0<\xi<\xi_{0} .
$$

Alors, selon (37), nous avons à considérer le problème suivant :

$$
L_{1} h \equiv \mu h^{\prime}(\xi)+E \geq 0, \quad \mu h(\xi)-1 \cdot h^{\prime}(\xi)=C_{0}, \quad 0<\xi<\xi_{0} .
$$

Ce problème admet pour solution particulière

$$
\begin{gathered}
h(\xi)=C_{1} \mathrm{e}^{\mu \xi}+\frac{C_{0}}{\mu}, \quad 0<\xi<\xi_{0}, \\
\text { avec } C_{1}=-\frac{E}{\mu^{2}} \mathrm{e}^{-\mu \xi_{0}}, \quad \frac{C_{0}}{\mu}=h_{0}(0)+\frac{E}{\mu^{2}} \mathrm{e}^{-\mu \xi_{0}}, \quad h_{0}(0)=h(0)>0, \quad 0<\xi<\xi_{0}, \\
\text { i.e. } h(\xi)=-\frac{E}{\mu^{2}} \mathrm{e}^{-\mu \xi_{0}} \mathrm{e}^{\mu \xi}+h_{0}(0)+\frac{E}{\mu^{2}} \mathrm{e}^{-\mu \xi_{0}}, \quad \lambda(\xi) \equiv 1, \quad 0<\xi<\xi_{0}, \\
h(\xi) \in] h_{0}(0)-\frac{E}{\mu^{2}}\left(1-\mathrm{e}^{-\mu \xi_{0}}\right), h_{0}\left[, \quad h_{0}(0)=h(0)>0, \quad 0<\xi<\xi_{0} .\right.
\end{gathered}
$$


Supposons par réalisme, pour que $h$ reste positif, que

$$
h_{0}(0)-\frac{E}{\mu^{2}}\left(1-\mathrm{e}^{-\mu \xi_{0}}\right) \geq h_{0}(0)-\frac{E}{\mu^{2}}>0 .
$$

Alors

$$
h(\xi) \in] h_{0}(0)-\frac{E}{\mu^{2}}\left(1-\mathrm{e}^{-\mu \xi_{0}}\right), h_{0}(0)[\subset] 0, h_{0}(0)[.
$$

Il est alors facile de vérifier que la solution sélectionnée vérifie précisément

$$
\begin{gathered}
L_{1}(h \mid \xi) \equiv \mu h^{\prime}(\xi)+E=\mu^{2} C_{1} \mathrm{e}^{\mu \xi}+E=E\left(1-\mathrm{e}^{-\mu \xi_{0}} \mathrm{e}^{\mu \xi}\right) \geq 0, \quad 0<\xi<\xi_{0}, \\
L_{1}\left(h \mid \xi_{0}\right) \equiv \mu h^{\prime}\left(\xi_{0}\right)+E=E(1-1)=0 .
\end{gathered}
$$

3.2.2. L'intervalle $] \xi_{0},+\infty[$

Supposons que sur l'intervalle contenu dans $] \xi_{0},+\infty[$, on ait à réaliser

$$
L_{1}(h \mid \xi) \equiv \mu h^{\prime}(\xi)+E^{*}=0, \quad L_{3} \lambda \equiv 1-\lambda(\xi) \geq 0, \quad \xi_{0}<\xi<+\infty .
$$

Alors, selon (37), nous avons à considérer le problème suivant

$$
L_{1}(h \mid \xi) \equiv \mu h^{\prime}(\xi)+E^{*}=0, \quad \mu h(\xi)-\lambda(\xi) h^{\prime}(\xi)=C_{0}=\mu h_{0}+\frac{E}{\mu} \mathrm{e}^{-\mu \xi_{0}} .
$$

Le problème (43) a une solution donnée par

$$
h(\xi)=-\frac{E^{*}}{\mu} \xi+C_{2}, \quad C_{2}=\frac{E^{*}}{\mu} \xi_{0}+h\left(\xi_{0}\right)
$$

i.e.

$$
\begin{gathered}
h(\xi)=-\frac{E^{*}}{\mu}\left(\xi-\xi_{0}\right)+h_{0}(0)-\frac{E}{\mu^{2}}\left(1-\mathrm{e}^{-\mu \xi_{0}}\right), \quad \lambda(\xi)=\mu \xi+\frac{E}{E^{*}}-\mu \xi_{0} . \\
L_{1}(h \mid \xi) \equiv \mu h^{\prime}(\xi)+E^{*}=0 .
\end{gathered}
$$

Ainsi, nous devons imposer la condition de cohérence

$$
\lambda(\xi)=\mu \xi+\frac{E}{E^{*}}-\mu \xi_{0} \leq 1, \quad \text { i.e. } \quad \xi_{1} \leq \xi_{0}+\frac{E^{*}-E}{\mu E^{*}} .
$$

Il est facile de vérifier que les conditions

$$
h\left(\xi_{0}-0\right)=-\frac{E}{\mu^{2}}+h_{0}+\frac{E}{\mu^{2}} \mathrm{e}^{-\mu \xi_{0}}=h\left(\xi_{0}+0\right)
$$

sont assurées. Soit alors (détermination du point le plus bas admissible)

$$
h\left(\xi_{1}\right)=\min _{\left[\xi_{0}, \xi_{1}\right]} h(\xi)=-\frac{E^{*}}{\mu}\left(\xi_{1}-\xi_{0}\right)+h_{0}(0)-\frac{E}{\mu^{2}}\left(1-\mathrm{e}^{-\mu \xi_{0}}\right)=0 .
$$


Il s'ensuit en vertu de (40) que

$$
\xi_{1}=\xi_{0}+\frac{\mu}{E^{*}}\left(h_{0}(0)-\frac{E}{\mu^{2}}\left(1-\mathrm{e}^{-\mu \xi_{0}}\right)\right) \geq \xi_{0}+\frac{\mu}{E^{*}}\left(h_{0}(0)-\frac{E}{\mu^{2}}\right)=\xi_{M}>\xi_{0} .
$$

La solution trouvée est donc définie sur l'intervalle $] 0, \xi_{1}\left[\right.$ avec $\xi_{1}=\min \left\{\xi_{M}, \xi_{0}+\frac{E^{*}-E}{\mu E^{*}}\right\}$ et dépend des constantes positives suivantes :

$$
h_{0}, E, E^{*}, \mu, \xi_{0},\left(h_{0}(0)-\frac{E}{\mu^{2}}\right)>0
$$

En résumé, prenant

$$
E(\xi)=E \chi_{] 0, \xi_{0}[}+E^{*} \chi_{] \xi_{0}, \xi_{1}[}, E^{*}>E, \quad \xi=x+\mu t
$$

on met en évidence le couple-solution (avec les notations et conditions précédentes)

$$
\begin{aligned}
& h(x, t)=-\frac{E}{\mu^{2}} \mathrm{e}^{-\mu \xi_{0}} \mathrm{e}^{\mu \xi}+h_{0}(0)+\frac{E}{\mu^{2}} \mathrm{e}^{-\mu \xi_{0}} ; \quad \lambda(x, t) \equiv 1, \quad 0<\xi \leq \xi_{0}, \\
& h(x, t)=-\frac{E^{*}}{\mu}\left(\xi-\xi_{0}\right)+h_{0}(0)-\frac{E}{\mu^{2}}\left(1-\mathrm{e}^{-\mu \xi_{0}}\right) ; \quad \lambda(x, t)=\mu\left(\xi-\xi_{0}\right)+\frac{E}{E^{*}}, \quad \xi_{0} \leq \xi \leq \xi_{1} .
\end{aligned}
$$

On observe à nouveau que la fonction $(t, x) \mapsto \lambda(t, x)$ est discontinue pour les valeurs de $(t, x)$ telles que $\xi_{0}=x+\mu t$ (voir que $\left.\lambda\left(\xi_{0}-0\right)=1, \lambda\left(\xi_{0}+0\right)=\frac{E}{E^{*}}<1\right)$, de même que pour la fonction $(t, x) \mapsto h^{\prime}(t, x)($ voir que $\left.h^{\prime}\left(\xi_{0}-0\right)=-\frac{E}{\mu}, h^{\prime}\left(\xi_{0}+0\right)=-\frac{E^{*}}{\mu}\right)$ alors que le produit $\lambda h^{\prime}$ est continu.

\subsection{Exemples de calcul (avec obstacle mobile)}

3.3.1. Variante 1 : érosion très faible, évolution lente, $\lambda$ discontinu

Les données numériques sont ici

$$
\begin{gathered}
h_{0}=h_{0}(0)=2, E=0,0001, \mu=0,01, E^{*}=0,0005, \xi_{0}=1, \xi_{1}=81, h_{0}-\frac{E}{\mu^{2}}>0 \\
\Omega=] 0,1\left[, \Gamma_{e}=\{x=0\}, \Gamma_{s}=\{x=1\}, \xi=x+\frac{1}{100} t,\right. \\
E(x, t)=0,0001 \chi_{\left\{0 \leq x+\frac{1}{100} t<1\right\}}+0,0005 \chi_{\left\{1 \leq x+\frac{1}{100} t \leq 21\right\}}, T=2000,
\end{gathered}
$$

de sorte que l'expression littérale trouvée précédemment conduit à la représentation explicite

$$
h(x, t)=\left\{\begin{array}{ccc}
-\mathrm{e}^{\frac{1}{100}\left(x+\frac{1}{100} t-1\right)}+2+\mathrm{e}^{-\frac{1}{100}} & \text { si } & 0<x+\frac{1}{100} t \leq 1 \\
-0,05\left(x+\frac{1}{100} t-1\right)+1+\mathrm{e}^{-\frac{1}{100}} & \text { si } & 1 \leq x+\frac{1}{100} t \leq 21 .
\end{array}\right.
$$


L'expression littérale du coefficient $\lambda$ (discontinu au début du phénomène) est donnée par

$$
\lambda(x, t)=\left\{\begin{array}{ccc}
1 & \text { si } & 0<x+\frac{1}{100} t<1 \\
\frac{1}{5}+\frac{1}{100}\left(x+\frac{1}{100} t-1\right) & \text { si } & 1 \leq x+\frac{1}{100} t \leq 21
\end{array}\right.
$$

en correspondance avec les données sur le bord parabolique suivantes :

$$
\left.q_{0}(t)=-\frac{1}{100} e^{\frac{1}{100}\left(\frac{1}{100} t-1\right)}, \quad t \in\right] 0,100\left[, \quad q_{0}(t)=-0,05\left(\frac{1}{5}+\frac{1}{100}\left(\frac{1}{100} t-1\right)\right), \quad t \geq 100,\right.
$$

$q_{1}$ étant assujettie à vérifier : $q_{1}(t) \geq 10^{-2}+510^{-6} t$,

$$
\left.h_{0}(x)=2+\mathrm{e}^{-\frac{1}{100}}-\mathrm{e}^{\frac{1}{100}(x-1)}, \quad x \in\right] 0,1[,
$$

de sorte que sont effectivement vérifiées les conditions d'asservissement imposée sur $\left.\Gamma_{s} \times\right] 0, T$.

Le graphe lipschitzien de la fonction $x \mapsto h(x, t)$, pour $t \in] 0,100[$, présente un point anguleux en $x=1-\frac{1}{100} t$, là où la fonction $x \mapsto \lambda(x, t)$ est discontinue :

$$
\lambda\left(\left(1-\frac{1}{100} t\right)^{-}, t\right)=1, \lambda\left(\left(1-\frac{1}{100} t\right)^{+}, t\right)=\frac{1}{5} .
$$

Après l'instant $t=100$, le graphe de la fonction $x \mapsto h(x, t), x \in[0,1]$, est une droite de pente $-0,05$, de point le plus haut à la cote $-0,05\left(\frac{1}{100} t-1\right)+1+\mathrm{e}^{-\frac{1}{100}}$ et la fonction $x \mapsto \lambda(x, t)$ est affine croissante. Le point le plus bas est à la cote $-\frac{5}{10000} t+1+\mathrm{e}^{-\frac{1}{100}}$, ce qui montre que le phénomène pourrait se poursuivre selon cette loi plus longtemps (jusqu'à l'instant $T$ tel que $\frac{5}{10000} T=1+\mathrm{e}^{-\frac{1}{100}}$, i.e. $\simeq 3980$ ), le paramètre $\lambda$ restant pour ces valeurs en-deçà de 1 .

3.3.2. Variante 2 : érosion très faible; $\lambda$ discontinu et s'annulant, un exemple non trivial de non-unicité Les données numériques rendant compte d'une région sans érosion sont ici

$$
\begin{gathered}
h_{0}=h_{0}(0)=100, E=0, \mu=0,001, E^{*}=0,0001, \xi_{0}=1, \xi_{1}=1001, h_{0}-\frac{E}{\mu^{2}}>0 . \\
\Omega=] 0,1\left[, \Gamma_{e}=\{x=0\}, \Gamma_{s}=\{x=1\}, \xi=x+\frac{1}{1000} t\right. \\
E(x, t)=0,0001 \chi_{\left\{1 \leq x+\frac{1}{1000} t \leq 1001\right\}}, \quad T=10^{6},
\end{gathered}
$$

de sorte que l'expression littérale trouvée précédemment conduit à la représentation explicite

$$
h(x, t)=\left\{\begin{array}{ccl}
100 & \text { si } & 0<x+\frac{1}{1000} t \leq 1 \\
-0,1\left(x+\frac{1}{1000} t-1\right)+100 & \text { si } & 1 \leq x+\frac{1}{1000} t \leq 1001
\end{array}\right.
$$


L'expression littérale du coefficient $\lambda$ (discontinu au début du phénomène) est donnée par

$$
\lambda(x, t)=\left\{\begin{array}{cll}
1 & \text { si } & 0<x+\frac{1}{1000} t<1 \\
\frac{1}{1000}\left(x+\frac{1}{1000} t-1\right) & \text { si } & 1 \leq x+\frac{1}{1000} t \leq 1001
\end{array}\right.
$$

Les expressions des flux de bord correspondants et de l'état initial sont précisées par les relations suivantes :

$$
\left.q_{0}(t)=0 \quad \text { pour } t \in\right] 0,1000\left[; \quad q_{0}(t)=-\frac{1}{10000}\left(\frac{1}{1000} t-1\right) \quad \text { pour } t \geq 1000,\right.
$$

$q_{1}$ étant uniquement assujettie à vérifier : $q_{1}(t) \geq 10^{-7} t, \quad t \geq 0$,

$$
\left.h_{0}(x)=100, \quad x \in\right] 0,1[,
$$

de sorte que sont effectivement vérifiées les conditions d'asservissement sur $\left.\Gamma_{s} \times\right] 0, T[$

$$
\begin{gathered}
\lambda(1, t) \partial_{x} h(1, t)+q_{1}(t) \geq 0, \quad \partial_{t} h(1, t)=-0,0001, \\
\left(\partial_{t} h(1, t)+0,0001\right)\left(\lambda(1, t) \partial_{x} h(1, t)+q_{1}(t)\right)=0 .
\end{gathered}
$$

Le graphe lipschitzien de la fonction $x \mapsto h(x, t)$, pour $t \in] 0,1000[$, présente un point anguleux mobile parcourant l'intervalle $[0,1]$ de droite à gauche en $x=1-\frac{1}{1000} t$, là où la fonction $x \mapsto \lambda(x, t)$ est discontinue et nulle à droite :

$$
\lambda\left(\left(1-\frac{1}{1000} t\right)^{-}, t\right)=1, \quad \lambda\left(\left(1-\frac{1}{1000} t\right)^{+}, t\right)=0 .
$$

Après l'instant $t=1000$, le graphe de la fonction $x \mapsto h(x, t), x \in[0,1]$, est une droite de pente $-0,1$, de point le plus haut à la cote $-0,1\left(\frac{1}{1000} t-1\right)+100$, de point le plus bas atteignant la cote limite 0 à l'instant $t=10^{6}$ et la fonction $x \mapsto \lambda(x, t)$ est affine croissante.

On se rend compte sur cet exemple que toute proposition de la forme

$$
h(x, t)=\left\{\begin{array}{cll}
100 & \text { si } & 0<x+\frac{1}{1000} t \leq 1 \\
-0,1\left(x+\frac{1}{1000} t-1\right)+100 & \text { si } & 1 \leq x+\frac{1}{1000} t \leq 1001
\end{array}\right.
$$

avec pour choix du coefficient $\lambda$ associé l'expression donnée par

$$
\lambda(x, t)=\left\{\begin{array}{ccc}
\text { toute fonction } \mathcal{L}^{2}-\text { mesurable à valeurs dans }[0,1] & \text { si } & 0<x+\frac{1}{1000} t<1 \\
\frac{1}{1000}\left(x+\frac{1}{1000} t-1\right) & \text { si } & 1 \leq x+\frac{1}{1000} t \leq 1001
\end{array}\right.
$$

constitue une solution du problème, pour les mêmes données, ce qui montre que le problème II n'est pas bien posé au sens de Hadamard (non-unicité du couple-solution). La solution exhibée en premier lieu représente une solution maximale dans la classe des solutions construites par ce procédé. 


\section{Approche thÉORIQUe du PROBLÈme PRÉPARATOIRE}

\subsection{Rappel des notations}

Rappelons brièvement les notations suivantes :

$\Omega$ représente un domaine régulier de $\mathbb{R}^{N}(N=1$ ou 2 dans notre étude), de frontière $\Gamma$ partagée en deux parties $\Gamma_{e}$ et $\Gamma_{s}$ et de normale extérieure notée $\vec{n}$.

Pour tout nombre positif $T$, on note $Q=] 0, T[\times \Omega, \Sigma=] 0, T\left[\times \Gamma, \Sigma_{e}=\right] 0, T\left[\times \Gamma_{e}\right.$ et $\left.\Sigma_{s}=\right] 0, T\left[\times \Gamma_{s}\right.$.

Le problème posé (par Eymard et al. [4] pour des modèles de géologies à l'I.F.P.) est donc de trouver un couple $(\lambda, h)$, a priori dans $L^{\infty}(Q) \times W^{1,2}\left(0, T ; H^{1}(\Omega)\right)$, vérifiant :

$$
\begin{aligned}
\partial_{t} h(t, x)-\operatorname{Div}\{\lambda(t, x) \nabla h(t, x)\} & =0 \quad \text { dans } Q, \\
h(0, x) & =h_{0} \quad \text { dans } \Omega \\
\lambda \partial_{n} h+f & =0 \quad \text { sur } \Sigma_{e} \\
\text { i) } \quad \lambda \partial_{n} h+f \geq 0, \quad \text { ii) } \quad \partial_{t} h+E & \geq 0 \quad \text { avec } \quad \text { iii }) \quad\left(\partial_{t} h+E\right)\left(\lambda \partial_{n} h+f\right)=0 \quad \text { sur } \Sigma_{S}, \\
\partial_{t} h+E & \geq 0 \quad \text { dans } Q, \\
a & \leq \lambda \leq 1 \text { où } a>0
\end{aligned}
$$

où $f$ et $E$ sont des fonctions régulières de $[0, T] \times \Gamma$ à valeurs dans $\mathbb{R}$ et $[0, T] \times \bar{\Omega}$ à valeurs dans $\mathbb{R}^{+}$respectivement.

Afin de simplifier l'écriture dans la suite, notons

$$
H=L^{2}(\Omega), \quad V=H^{1}(\Omega), \quad K=\left\{v \in L^{2}(0, T ; V), v+E \geq 0 \operatorname{sur} \Sigma_{s}\right\}
$$

et pour tout $u$ et $v$ de $V$,

$$
a_{\lambda}(u, v)=\int_{\Omega} \lambda(t, x) \nabla u \nabla v \mathrm{~d} x
$$

Dans le cadre de notre présentation, le problème complet : trouver $(\lambda, h)$ solution de (45) à (50) est ouvert. Aussi, nous envisagerons l'étude suivante (problème préparatoire pour une donnée de $\lambda$, présentant $a$ priori à $t$ fixé des discontinuités selon les indications des géologues, mais évoluant « régulièrement ») :

soient $a>0$ et $\lambda$ fixé dans $W^{1, \infty}\left(0, T ; L^{\infty}(\Omega)\right)$ tels que $1 \geq \lambda(t,) \geq a>$.0 p.p. dans $\Omega$; existe-t-il une solution unique en son genre $h$ satisfaisant les relations (45) à (49)?

\subsection{Formulation variationnelle $\mathbf{d u}$ problème}

En s'appuyant sur les travaux de G. Duvaut et J.-L. Lions [3] (Chap. 2), concernant les modèles d'asservissement thermique (voir aussi les « nouveaux problèmes unilatéraux » de J.-L. Lions [8] p. 420 et passim), on 
dira que $h$ est une solution de $(45)$ à $(49)$, si

$$
\begin{gathered}
h \in W^{1,2}(0, T ; V) \text { avec } \partial_{t} h \in K \\
\left.h(0)=h_{0} \quad p . p . \text { dans } \Omega \quad \text { et } \quad \forall v \in V, t p \cdot p . \text { dans }\right] 0, T[, \\
\int_{\Omega} \partial_{t} h\left(v-\partial_{t} h\right) \mathrm{d} x+a_{\lambda}\left(h, v-\partial_{t} h\right)+\int_{\Gamma} f\left(v-\partial_{t} h\right) \mathrm{d} \sigma+\int_{\Gamma_{s}} \chi_{\mathbb{R}^{+}}(v+E) \mathrm{d} \sigma \geq 0
\end{gathered}
$$

en notant $\chi_{\mathbb{R}^{+}}(x)=\left\{\begin{array}{l}0 \text { si } x \geq 0 \\ +\infty \text { si } x<0\end{array}\right.$.

On retrouve alors l'équation (45) en l'interprétant dans un premier temps au sens des distributions, puis dans $L^{2}$ et presque partout dans $Q$.

Les conditions de bord (47) et (48), vérifiées formellement dans $\Sigma_{e}$ et $\Sigma_{s}$, se trouvent alors justifiées au sens de $H_{00}^{-\frac{1}{2}}\left(\Gamma_{e}\right)$ et $H_{00}^{-\frac{1}{2}}\left(\Gamma_{s}\right)$ respectivement.

\subsection{Commentaires sur la donnée initiale}

Le choix de la donnée initiale est primordial (nous renvoyons le lecteur à la Sect. 2.1 pour s'en convaincre). Dans le cadre de cette étude mathématique, il nous faut choisir $h_{0}$ compatible avec la contrainte (49); c'est-à-dire spéculer sur la valeur de $h_{1}=\operatorname{Div}\left(\lambda(0,.) \nabla h_{0}\right)$ pour satisfaire $h_{1}+E(0) \geq 0$ p.p. dans $\Omega$.

Ainsi, dans la suite on suppose que :

$$
\int_{\Gamma} f(0) \mathrm{d} \sigma<\int_{\Omega} E(0) \mathrm{d} x
$$

et que $h_{0}$ est une solution dans $V$ (à une constante additive près) du problème de Neumann :

$$
\begin{aligned}
\operatorname{Div}(\lambda(0, x) \nabla u) & =g-E(0)=h_{1} \quad \operatorname{dans} \Omega, \\
-\lambda(0, x) \partial_{n} u & =f(0) \quad \text { sur } \Gamma,
\end{aligned}
$$

où $g$ est une fonction positive de $H$ telle que $\int_{\Omega} g \mathrm{~d} x=\int_{\Omega} E(0) \mathrm{d} x-\int_{\Gamma} f(0) \mathrm{d} \sigma$.

\subsection{Existence d'une solution}

Pour cela, nous allons adapter au cas d'une famille de formes bilinéaires dépendant du temps la méthode présentée par G. Duvaut et J.-L. Lions dans [3] et J.-L. Lions [8], reposant sur une pénalisation de la contrainte, une régularisation hyperbolique d'ordre deux en temps pour le traitement d'un problème a priori singulier (le taux d'érosion $\partial_{t} h$ apparaît à la fois dans $\Omega$ et sur une partie de $\partial \Omega$ ), puis une méthode de Galerkin.

Introduisons alors, pour tout $\eta$ strictement positif :

- la fonction numérique $\beta_{\eta}$, définie pour $x$ réel par $\beta_{\eta}(x)=\frac{1}{\eta} \beta(x)$, où, $\beta(x)=-x \mathbb{I}_{]-\infty,-1[}(x)+x\left(x^{2}+\right.$ 2) $\mathbb{I}_{[-1,0]}(x)$;

- $\lambda_{\eta}$ une suite d'éléments de $W^{2, \infty}\left(0, T ; L^{\infty}(Q)\right)$ qui converge vers $\lambda$ dans $W^{1, \infty}\left(0, T ; L^{\infty}(Q)\right)$ et vérifiant aussi $\lambda_{\eta}(t,) \geq a>$.0 p.p. dans $\Omega$;

- $g_{\eta}$ une suite d'éléments de $V$ qui converge dans $H$ vers $g$ (présentée ci-dessus). De sorte que $h_{1 \eta}=g_{\eta}-E(0)$ converge vers $h_{1}$ dans $H$ et il existe une suite $h_{0 n}$ de solutions du problème (52), pour le second membre $h_{\eta 1}$, qui converge dans $V$ vers $h_{0}$ (voir modulo une suite numérique par stabilité du problème dans $H^{1}(\Omega) / \mathbb{R}$ ).

Considérons une base hilbertienne $\left(e_{i}\right)_{i \in \mathbb{N}^{*}} \operatorname{de} V$; notons $V_{n}=\operatorname{Vect}\left[e_{1}, \ldots, e_{n}\right]$ et $\pi_{n}$ la projection orthogonale de $V$ sur $V_{n}$. 
On admet enfin que pour tout entier $n$ supérieur à $3, h_{0}, h_{1}$ et $E(0)$ sont des éléments de $V_{n}$.

Commençons par la première proposition, relative à la méthode de Galerkin.

Proposition 4.1. Pour tout paramètre strictement positif $\varepsilon$ et $\eta$ et tout entier $n$, il existe une unique solution au problème $\left(P_{\eta, \varepsilon}^{n}\right)$ suivant :

$$
\begin{gathered}
h_{\eta, \varepsilon}^{n}, \partial_{t} h_{\eta, \varepsilon}^{n}, \partial_{t}^{2} h_{\eta, \varepsilon}^{n} \in L^{2}\left(0, T ; V_{n}\right) \\
h_{\eta, \varepsilon}^{n}(0)=h_{0 \eta} p . p . \text { dans } \Omega, \partial_{t} h_{\eta, \varepsilon}^{n}(0)=h_{1 \eta} p . p . \text { dans } \Omega \quad \text { et } \quad \forall v \in V_{n}, \\
\varepsilon \int_{\Omega} \partial_{t}^{2} h_{\eta, \varepsilon}^{n} v \mathrm{~d} x+\int_{\Omega} \partial_{t} h_{\eta, \varepsilon}^{n} v \mathrm{~d} x+a_{\lambda_{\eta}}\left(h_{\eta, \varepsilon}^{n}, v\right)+\int_{\Gamma} f v \mathrm{~d} \sigma-\int_{\Gamma_{s}} \beta_{\eta}\left[\partial_{t} h_{\eta, \varepsilon}^{n}+E_{n}\right] v \mathrm{~d} \sigma=0 \quad t p . p .
\end{gathered}
$$

où on note $E_{n}=\pi_{n}(E)$. De plus, $h_{\eta, \varepsilon}^{n} \in W^{3, \infty}\left(0, T ; V_{n}\right)$ et pour tout $v$ de $V_{n}$,

$$
\begin{gathered}
\varepsilon \int_{\Omega} \partial_{t}^{3} h_{\eta, \varepsilon}^{n} v \mathrm{~d} x+\int_{\Omega} \partial_{t}^{2} h_{\eta, \varepsilon}^{n} v \mathrm{~d} x+a_{\lambda_{\eta}}\left(\partial_{t} h_{\eta, \varepsilon}^{n}, v\right)+a_{\partial_{t} \lambda_{\eta}}\left(h_{\eta, \varepsilon}^{n}, v\right) \\
+\int_{\Gamma} \partial_{t} f v \mathrm{~d} \sigma-\frac{1}{\eta} \int_{\Gamma_{s}} \beta_{\eta}^{\prime}\left(\partial_{t} h_{\eta, \varepsilon}^{n}+E_{n}\right)\left(\partial_{t}^{2} h_{\eta, \varepsilon}^{n}+\partial_{t} E_{n}\right) v \mathrm{~d} \sigma=0 \quad t p . p .
\end{gathered}
$$

C'est un résultat classique d'équation différentielle ordinaire lié à la régularité de $\beta$.

Dès lors, nous devons mettre en évidence l'existence d'une solution au problème hyperbolique d'ordre deux $\left(P_{\eta, \varepsilon}\right)$ suivant :

$$
\begin{gathered}
h_{\eta, \varepsilon} \in L^{2}(0, T ; V), \partial_{t} h_{\eta, \varepsilon} \in L^{2}(0, T ; V) \text { et } \partial_{t}^{2} h_{\eta, \varepsilon} \in L^{2}(0, T ; H) \\
h_{\eta, \varepsilon}(0)=h_{0 \eta} p . p . \text { dans } \Omega, \partial_{t} h_{\eta, \varepsilon}(0)=h_{1 \eta} p . p . \text { dans } \Omega \text { et } \forall v \in V \\
\varepsilon \int_{\Omega} \partial_{t}^{2} h_{\eta, \varepsilon} v \mathrm{~d} x+\int_{\Omega} \partial_{t} h_{\eta, \varepsilon} v \mathrm{~d} x+a_{\lambda_{\eta}}\left(h_{\eta, \varepsilon}, v\right)+\int_{\Gamma} f v \mathrm{~d} \sigma-\int_{\Gamma_{s}} \beta_{\eta}\left(\partial_{t} h_{\eta, \varepsilon}+E\right) v \mathrm{~d} \sigma=0 \quad t p . p .
\end{gathered}
$$

Dans cette démarche, une série d'estimations a priori est nécessaire et fait l'objet des lemmes suivants.

Lemme 4.2. Il existe une constante strictement positive $M_{1}$, dépendant de $\left\|h_{0}\right\|_{V},\left\|h_{1}\right\|_{H},\|\lambda\|_{\infty}$ et $\left\|\partial_{t} \lambda\right\|_{\infty}$, telle que pour tout $t$,

$$
\left\|\sqrt{\varepsilon} \partial_{t} h_{\eta, \varepsilon}^{n}(t)\right\|_{H}+\left\|\partial_{t} h_{\eta, \varepsilon}^{n}\right\|_{L^{2}(0, t ; H)}+\left\|h_{\eta, \varepsilon}^{n}(t)\right\|_{V}+\left\|\frac{1}{\eta} g\left[\partial_{t} h_{\eta, \varepsilon}^{n}+E_{n}\right]\right\|_{L^{1}(0, t ; H)} \leq M_{1}
$$

où on note $g(x)=-\beta(x) x$.

La justification de ce lemme découle de l'emploi de la fonction test $v=\partial_{t} h_{\eta, \varepsilon}^{n}$ dans le problème $\left(P_{\eta, \varepsilon}^{n}\right)$. 
Lemme 4.3. Il existe une constante strictement positive $M_{2}$, dépendant de $\left\|h_{0}\right\|_{V},\left\|h_{1 \eta}\right\|_{V},\|\lambda\|_{\infty},\left\|\partial_{t} \lambda\right\|_{\infty}$ et $\left\|\partial_{t}^{2} \lambda_{\eta}\right\|_{\infty}$, telle que pour tout $t$

$$
\left\|\sqrt{\varepsilon} \partial_{t}^{2} h_{\eta, \varepsilon}^{n}(t)\right\|_{H}+\left\|\partial_{t}^{2} h_{\eta, \varepsilon}^{n}\right\|_{L^{2}(0, t ; H)}+\left\|\partial_{t} h_{\eta, \varepsilon}^{n}(t)\right\|_{V} \leq M_{2}
$$

Pour démontrer ce lemme, il suffit de poser $v=\partial_{t}^{2} h_{\eta, \varepsilon}^{n}+\partial_{t} E_{n}$ dans (54), en remarquant que le choix particularisé de $h_{1 \eta}$ conduit à $\partial_{t}^{2} h_{\eta, \varepsilon}^{n}(0)=0$.

Lemme 4.4. Il existe une constante strictement positive $M_{3}$, dépendant de $\left\|h_{0}\right\|_{V},\left\|h_{1}\right\|_{H}$, $\|\lambda\|_{\infty}$ et $\left\|\partial_{t} \lambda\right\| \|_{\infty}$, telle que pour tout $t$,

$$
\left\|\partial_{t} h_{\eta, \varepsilon}^{n}(t)\right\|+\left\|\partial_{t} h_{\eta, \varepsilon}^{n}\right\|_{L^{2}(0, t ; V)} \leq M_{3}\left(1+M_{2} \sqrt{\varepsilon}\right)
$$

Il suffit d'utiliser $v=\partial_{t} h_{\eta, \varepsilon}^{n}+E_{n}$ dans (54) et la preuve résulte de manipulations algébriques élémentaires.

Dès lors, suivant J.-L. Lions [8], J.-L. Lions et W. Strauss [11] ou encore G. Duvaut et J.-L. Lions [3], il est possible de passer à la limite sur $n$, puis $\varepsilon$, et de mettre en évidence que :

Proposition 4.5. Il existe une unique solution au problème pénalisé $\left(P_{\eta}\right)$ suivant :

$$
\begin{gathered}
h_{\eta} \in L^{2}(0, T ; V), \partial_{t} h_{\eta} \in L^{2}(0, T ; V) \text { avec } \partial_{t}^{2} h_{\eta} \in L^{2}(0, T ; H) \\
h_{\eta}(0)=h_{0 \eta} p . p . \text { dans } \Omega \quad \text { et } \quad \forall v \in V, \\
\int_{\Omega} \partial_{t} h_{\eta} v \mathrm{~d} x+a_{\lambda_{\eta}}\left(h_{\eta}, v\right)+\int_{\Gamma} f v \mathrm{~d} \sigma-\int_{\Gamma_{s}} \beta_{\eta}\left(\partial_{t} h_{\eta}+E\right) v \mathrm{~d} \sigma=0 \quad t p . p .
\end{gathered}
$$

De plus, il existe une constante strictement positive $M_{4}$, dépendant de $\left\|h_{0}\right\|_{V},\left\|h_{1}\right\|_{H},\|\lambda\|_{\infty}$ et $\left\|\partial_{t} \lambda\right\|_{\infty}$, telle que

$$
\left\|h_{\eta}\right\|_{L^{\infty}(0, T ; V)}+\left\|\frac{1}{\eta} g\left[\partial_{t} h_{\eta}+E\right]\right\|_{L^{1}(Q)}+\left\|\partial_{t} h_{\eta}\right\|_{L^{\infty}(0, T ; H)}+\left\|\partial_{t} h_{\eta}\right\|_{L^{2}(0, T ; V)} \leq M_{4} .
$$

De plus, $\left\|\partial_{t}^{2} h_{\eta}\right\|_{L^{2}\left(0, T ; V_{0}^{\prime}\right)} \leq M_{4}$ ò̀ $V_{0}=\left\{v \in V, v=0\right.$ sur $\left.\Gamma_{s}\right\}$.

La dernière majoration découle du fait que pour tout $v$ de $V$, nul sur $\Gamma_{s}$, on a

$$
\int_{\Omega} \partial_{t} h_{\eta} v \mathrm{~d} x+a_{\lambda_{\eta}}\left(h_{\eta}, v\right)+\int_{\Gamma} f v \mathrm{~d} \sigma=0
$$

Ainsi, la régularité affichée de $h_{\eta}$ conduit au résultat, via la relation :

$$
\int_{\Omega} \partial_{t}^{2} h_{\eta} v \mathrm{~d} x+a_{\lambda_{\eta}}\left(\partial_{t} h_{\eta}, v\right)+a_{\partial_{t} \lambda_{\eta}}\left(h_{\eta}, v\right)+\int_{\Gamma} \partial_{t} f v \mathrm{~d} \sigma=0
$$

Pour justifier l'existence d'une solution au problème (45) à (48), il nous reste alors à poser $v-\partial_{t} h_{\eta}$ comme fonction-test dans $\left(P_{\eta}\right)$ et à passer à la limite sur le paramètre $\eta$. Pour cela, les estimations de la précédente proposition fournissent les arguments de compacité (faible et forte) suffisants pour passer à la limite dans les premier et troisième termes de l'équation; le terme de pénalisation remplit parfaitement son rôle (grâce à la convexité de la fonction $g$ ) ; reste alors le deuxième terme de (56) (et plus particulièrement $a_{\lambda_{\eta}}\left(h_{\eta}, \partial_{t} h_{\eta}\right)$ ). 
Pour cela, il suffit alors de multiplier $\left(P_{\eta}\right)$ par $\mathrm{e}^{-\alpha t}$ où $\alpha=\sup _{n} \frac{\left\|\partial_{t} \lambda_{\eta}\right\|_{\infty}}{a}$ et de remarquer qu'alors

$$
\begin{aligned}
-\int_{0}^{T} a_{\lambda_{\eta}}\left(h_{\eta}, \partial_{t} h_{\eta}\right) \mathrm{e}^{-\alpha t} \mathrm{~d} t= & \frac{-1}{2} a_{\lambda_{\eta}(T)}\left(h_{\eta}(T), h_{\eta}(T)\right) \mathrm{e}^{-\alpha T}+\frac{1}{2} a_{\lambda_{\eta}(0)}\left(h_{0}, h_{0}\right) \\
& +\frac{1}{2} \int_{0}^{T}\left[a_{\partial_{t} \lambda_{\eta}}\left(h_{\eta}, h_{\eta}\right)-\alpha a_{\lambda_{\eta}}\left(h_{\eta}, h_{\eta}\right)\right] \mathrm{e}^{-\alpha t} \mathrm{~d} t
\end{aligned}
$$

conduit bien à la forme bilinéaire négative ( $c f$. J.-L. Lions [8], p. 402 ou encore J.-L. Lions et E. Magenes [9], Vol. 3, p. 109) recherchée, permettant de conclure :

Théorème 4.6. Il existe une unique solution de (51), i.e. de (45-47) et (48).

De plus, pour tout $v$ de $V_{0}$ et pour presque tout $t$,

$$
\left\langle\partial_{t}^{2} h, v\right\rangle_{V_{0}^{\prime}, V_{0}}+a_{\lambda}\left(\partial_{t} h, v\right)+a_{\partial_{t} \lambda}(h, v)+\int_{\Gamma} \partial_{t} f v \mathrm{~d} \sigma=0
$$

L'existence se déduit du passage à la limite décrit à l'instant. L'unicité se traite de façon classique en n'oubliant pas, pour une raison de monotonie, d'utiliser de nouveau la fonction $t \mapsto \mathrm{e}^{-\frac{\left\|\partial_{t} \lambda\right\| \|_{\infty}}{a} t}$.

\subsection{Au sujet de la positivité de $\partial_{t} h+E$ dans $Q$}

Donnons ici une condition suffisante de positivité dans $Q$ de $\partial_{t} h+E$.

Proposition 4.7. Si $\operatorname{div}\left(\partial_{t} \lambda \nabla h\right)+\partial_{t} E-\operatorname{div}(\lambda \nabla E) \geq 0$ dans $Q$ et $\partial_{t} f-\partial_{t} \lambda \partial_{n} h+\lambda \partial_{n} E \geq 0$ sur $\Sigma_{e}$ au sens où pour tout $\varphi$ positif de $L^{2}\left(0, T, V_{0}\right)$,

$$
\int_{0}^{t} \int_{\Omega} \partial_{t} E \varphi \mathrm{d} x \mathrm{~d} s+\int_{0}^{t} a_{\lambda}(E, \varphi) \mathrm{d} s-\int_{0}^{t} a_{\partial_{t} \lambda}(h, \varphi) \mathrm{d} s-\int_{\Sigma_{e}} \partial_{t} f \varphi \mathrm{d} \sigma \mathrm{d} s \geq 0,
$$

alors $\partial_{t} h+E \geq 0$ p.p. dans $Q$.

Cette proposition se justifie par une technique classique de principe du maximum.

À titre d'exemple (cf. Antontsev et al. [1]), si $E(t, x)=E_{0}, f(t, x)=f(x), K(t, x)=K(x)$ et $\lambda(t, x)=\lambda(x)$, alors $\partial_{t} h+E \geq 0$ p.p. dans $Q$.

\subsection{Conclusion et problèmes ouverts}

Sauf cas particuliers, l'étude théorique de l'existence et de l'unicité d'un couple $(\lambda, h)$ répondant aux problèmes I ou II reste encore un problème ouvert. Cela repose principalement sur la gageure de concilier la dualité entre une bonne régularité en temps nécessaire pour $\lambda$ afin d'obtenir une solution forte $h$ (puisque nous devons prendre en compte la trace de $\partial_{t} h$ sur une partie du bord du domaine) et le fait qu'aucune équation ne vient par son écriture faciliter cette exigence. Il manque alors des renseignements d'ordre physique pour qualifier $\lambda$, d'où la condition de maximalité du problème I.

L'obtention de solutions maximales pourrait être envisagée par le biais du lemme de Zorn. Toutefois, outre la difficulté technique de la mise en place d'une telle méthode, cette dernière approche ne fournirait qu'une solution maximale et non une borne maximale à l'ensemble des solutions. De plus, cette approche non constructive ne conduit guère à des idées de mise en œuvre de méthodes numériques.

Le problème II est d'une formulation plus aisée pour le calcul scientifique. Toutefois, ce dernier étant visiblement mal posé au regard de l'unicité de la solution, une condition de maximalité doit peut-être y être adjointe. 
On retrouve les soucis présentés ci-dessus, pour parvenir à une formulation bien posée au sens de Hadamard : dès lors que l'intuition et l'expérience du géologue portent à penser que le coefficient $\lambda$ présente, a priori, des discontinuités, il s'ensuit que le problème posé doit être alors considéré dans une formulation ultra-faible ( $c f$. sur ce point Brézis [2]) en recherchant $\lambda$ dans un ensemble admissible, contenant par exemple $L^{\infty}(Q) \cap \overline{B V}(Q)$.

\section{RÉFÉRENCES}

[1] S.N. Antontsev et G. Gagneux, Quelques remarques sur la recherche de la solution maximale d'un problème inverse en géologie, Publications du Laboratoire de Mathématiques Appliquées, ERS-CNRS 2055. Université de Pau, Analyse non linéaire, 2000/17 (2000).

[2] H. Brézis, Problèmes unilatéraux. J. Math. Pures Appl. IX (1972) 1-168.

[3] G. Duvaut et J.-L. Lions, Les inéquations en Mécanique et en Physique. Dunod, Paris (1972).

[4] R. Eymard, T. Gallouët, D. Granjeon, R. Masson et Q.H. Tran, Multi-lithology stratigraphic model under maximum erosion rate constraint. Int. J. Num. Methods Engrg. (soumis).

[5] D. Granjean, Q. Huy Tran, R. Masson et R. Glowinski, Modèle de sédimentation de bassins (en préparation) et Modèle stratigraphique multilithologique sous contrainte de taux d'érosion maximum. Document interne de l'Institut Français du Pétrole (2001).

[6] K. Ito et K. Kunisch, On the injectivity and linearization of the coefficient-to-solution mapping for elliptic boundary value problem. J. Math. Anal. Appl. 180 (1994) 1040-1066.

[7] J.-L. Lions, Remarks on evolution inequalities. J. Math. Soc. Japan 18 (1966) 331-342.

[8] J.-L. Lions, Quelques méthodes de résolution des problèmes aux limites non linéaires. Dunod, Paris (1969).

[9] J.-L. Lions et E. Magenes, Problèmes aux limites non homogènes et applications, Vols. I, II \& III. Dunod, Paris (1968).

[10] J.-L. Lions et G. Stampacchia, Variational inequalities. Comm. Pure Appl. Math. 20 (1967) 493-519.

[11] J.-L. Lions et W. Strauss, Some nonlinear evolution equations. Bull. Soc. Math. France 93 (1965) 43-96.

[12] S. Perez, Identification et homogénéisation de paramètres dans des équations aux dérivées partielles. Thèse de l'Université de Pau (1999).

[13] G.R. Richter, An inverse problem for the steady state diffusion equation. SIAM J. Appl. Math. 41 (1981) $210-221$.

[14] L. Schwartz, Théorie des distributions. Hermann, Paris (1966). 